\title{
Triaxial Accelerometer Error Coefficients Identification with a Novel Artificial Fish Swarm Algorithm
}

\author{
Yanbin Gao, ${ }^{1}$ Lianwu Guan, ${ }^{1,2}$ and Tingjun Wang ${ }^{3}$ \\ ${ }^{1}$ Institute of Inertial Navigation and Measurement \& Control Technology, College of Automation, Harbin Engineering University, \\ Harbin, Heilongjiang 150001, China \\ ${ }^{2}$ Navigation and Instrumentation Research Group (NavINST), Department of Electrical and Computer Engineering, \\ Queen's University, Kingston, ON, Canada K7L 3N6 \\ ${ }^{3}$ China Aerospace Science and Technology Corporation, No. 16, Xian, Shanxi 710001, China \\ Correspondence should be addressed to Lianwu Guan; guanlianwu@hrbeu.edu.cn
}

Received 4 March 2015; Accepted 13 May 2015

Academic Editor: Jong-Jae Lee

Copyright (C) 2015 Yanbin Gao et al. This is an open access article distributed under the Creative Commons Attribution License, which permits unrestricted use, distribution, and reproduction in any medium, provided the original work is properly cited.

Artificial fish swarm algorithm (AFSA) is one of the state-of-the-art swarm intelligence techniques, which is widely utilized for optimization purposes. Triaxial accelerometer error coefficients are relatively unstable with the environmental disturbances and aging of the instrument. Therefore, identifying triaxial accelerometer error coefficients accurately and being with lower costs are of great importance to improve the overall performance of triaxial accelerometer-based strapdown inertial navigation system (SINS). In this study, a novel artificial fish swarm algorithm (NAFSA) that eliminated the demerits (lack of using artificial fishes' previous experiences, lack of existing balance between exploration and exploitation, and high computational cost) of AFSA is introduced at first. In NAFSA, functional behaviors and overall procedure of AFSA have been improved with some parameters variations. Second, a hybrid accelerometer error coefficients identification algorithm has been proposed based on NAFSA and Monte Carlo simulation (MCS) approaches. This combination leads to maximum utilization of the involved approaches for triaxial accelerometer error coefficients identification. Furthermore, the NAFSA-identified coefficients are testified with 24-position verification experiment and triaxial accelerometer-based SINS navigation experiment. The priorities of MCS-NAFSA are compared with that of conventional calibration method and optimal AFSA. Finally, both experiments results demonstrate high efficiency of MCS-NAFSA on triaxial accelerometer error coefficients identification.

\section{Introduction}

Artificial fish swarm algorithm (AFSA) is one of the state-ofthe-art swarm intelligence approaches, which was proposed by Li et al. [1]. AFSA is inspired by the autonomous collective movement of the fishes and their various social behaviors. It has characteristics of global search, quick convergence rate, and efficient search based on modern elicitation methods. After the AFSA appears, it offers new ideas to solve the optimization problems in signal processing $[2,3]$, neural network classifiers $[4,5]$, data mining and clustering $[6,7]$, multiobjective optimization [8], PID controller parameters optimization [9], and so forth.

Nevertheless, the standard AFSA (SAFSA) has not been extensively considered by researchers, due to its complexity in comparison with other swarm intelligence algorithms in this domain. Particularly, concerning particle swarm optimization (PSO), the results of SAFSA are not better than those of PSO [10]. PSO is another swarm intelligence algorithm that simulates natural evolutionary processes to solve complex optimization problems. It has been successfully utilized in optimization problems, such as the multidimensional knapsack problem, the economic and economic statical designs, and the complex network reliability problem [11-16]. However, the reasons for SAFSA's inefficiency are high structural and computational complexities, lack of using previous experiences of the swarm individuals, and lack of appropriate balance between exploration and exploitation during optimization process [17-20]. According to these issues, a novel AFSA, called NAFSA, is introduced to conquer 
the weaknesses of SAFSA. For this purpose, different stages of AFSA are modified to eliminate the demerits and thus improve the efficiency of AFSA. The modifications include reducing the structural complexity as well as the computational complexity of SAFSA, determining a balance between the exploration and exploitation during optimization process, and adopting previous experiences of the swarm individuals to improve the optimization performance.

Triaxial accelerometer is widely utilized in military and civilian fields [21]. It is used for measuring the carrier's acceleration in three-dimensional (3D) space. Meanwhile, combined with the angular rate sensor-gyroscope, accelerometerbased strapdown inertial navigation system (SINS) could calculate high-resolution 3D navigation information (position, velocity, and attitude) $[22,23]$. However, the accelerometer error coefficients have to be identified accurately before it is adopted in SINS mechanization. Otherwise, the overall performance of accelerometer-based SINS would deteriorate greatly. So it is of great importance to calibrate the accelerometer error coefficients accurately $[24,25]$. Traditionally, highprecision turntable, which is relatively expensive for civilian fields, is utilized to identify the accelerometer error parameters. Therefore, study on the accelerometer error coefficients without the aid of turntable becomes a hot research topic in recent years.

At present, the optimal AFSA (OAFSA) with improvement on Visual and Step parameters has been utilized for fiber optical gyroscope random drift modeling by Wang et al. [26]. Meanwhile, the OAFSA is used for the real-time ring laser gyroscope bias temperature error compensation by $\mathrm{Yu}$ et al. [27]. Moreover, Gao et al. [18-20] have successfully adopted the OAFSA to calibrate the error parameters of accelerometer and fiber optical gyroscope (FOG) and verified the feasibility of the OAFSA on the accelerometer and FOG error coefficients recalibration. However, the OAFSA only makes the modification on the artificial fishes (AFs) Visual and Step parameters to balance the exploration and exploitation during optimization process and also adopts the secondary initialization method after certain times of AFSA optimization manually. But the structural and computational complexities of the algorithm remain and the AFs previous experiences are not utilized for improving the convergence rate. Therefore, solving these issues and letting the NAFSA recalibrate the accelerometer error coefficients are of great importance to improve the overall navigation performance of accelerometer-based SINS.

Monte Carlo simulation (MCS) is a broad class of computational algorithms that relies on repeated random sampling to obtain numerical results [28]. With MCS method, the computational results are closer to real conditions. So we adopt the MCS method to increase the credibility of the accelerometer error coefficients, which are optimized by NAFSA. Moreover, it also has the priority of reducing workload and costs over conventional high-precision turntable calibration method. Therefore, the hybrid MCSNAFSA technique that is based on MCS and NAFSA is the main contribution to the research on the recalibration of the accelerometer error parameters.
In conclusion, the advantages of the MCS-NAFSA for accelerometer error parameters identification are (1) that the algorithm's computational complexity is decreased to release the high computational cost, (2) that the algorithm's convergence rate is improved by adopting AFs' previous experiences during AFs optimization process, (3) that no external reference information is introduced during the identification process, and (4) that the high workload and costs in conventional calibration method are reduced greatly.

The rest of this paper is organized as follows. In Section 2, the SAFSA and its disadvantages on triaxial accelerometer error parameters identification are presented. Section 3 is briefly dedicated to the OAFSA and the secondary initialization method on triaxial accelerometer error parameters identification. The NAFSA's parameters and behaviors are described in detail in Section 4. Section 5 explains the triaxial accelerometer error parameters with MCS-NAFSA implementation procedures. After that, the simulation of triaxial accelerometer error parameters by MCS-NAFSA is conducted, and the results are discussed in Section 6. Next, the static 24-position triaxial accelerometer verification experiment and the accelerometer-based SINS navigation experiment with accelerometer error parameters identified by MCS-NAFSA are demonstrated in Section 7. Section 8 concludes this paper.

\section{Standard Artificial Fish Swarm Algorithm (SAFSA)}

2.1. Parameters of SAFSA. AFSA is one of the swarm intelligence methodologies and evolutionary optimization techniques and its framework is based on functions that are inspired by social behaviors of fishes in the nature. Generally, fishes move to the areas that have more food by AFs individual or swarm search. AF model is depicted by prey, swarm, free move, and following behaviors. AFs food consistency degree in a specific area is the AFSA objective function. Finally, the AFs approach the maximum food density point.

The state of AF $i$ is denoted as vector $X=\left(x_{1}, x_{2}, \ldots, x_{n}\right)$, and $x_{i}(i=1,2, \ldots, n)$ are the optimization variables. Current food consistency degree of AF $i$ in position $X$ can be expressed as objective function $Y=f(X)$. Visual is the sight field of AFs and Step denotes the maximum length of each AF movement. The distance between two AFs in $X_{i}$ and $X_{j}$ positions is represented by Euclidean Distance $\operatorname{Dis}_{i j}=$ $\left|X_{i}-X_{j}\right|$. Moreover, the best AF position is loaded in bulletin and crowd factor $\delta(0<\delta<1)$ is AFs' crowd degree within their Visual range. In the following subsection, behaviors of AFs will be described in detail.

\subsection{Behaviors of SAFSA}

2.2.1. Prey Behavior. In nature, fishes search for food or position with more food. Normally, we choose the position $X_{j}$ that is within the Visual range of AF $i$ randomly. Then, position $X_{j}$ is calculated by

$$
X_{i}(t+1)=X_{i}(t)+\operatorname{Visual} \times \operatorname{Rand}(-1,1) .
$$


Afterward, the food consistency degree $f(X)$ in positions $X_{i}$ and $X_{j}$ is calculated, respectively. If $f\left(X_{j}\right)>f\left(X_{i}\right), \mathrm{AF} i$ moves one Step forward from its current position $X_{i}$ towards $X_{j}$, which is performed by

$$
\begin{aligned}
X_{i}(t+1)= & X_{i}(t)+\frac{X_{j}(t)-X_{i}(t)}{\operatorname{Dis}_{i, j}} \\
& \times[\operatorname{Step} \times \operatorname{Rand}(0,1)],
\end{aligned}
$$

where $X_{i}$ is a $D$-dimensional vector and $\vec{X}_{j}-\vec{X}_{i}$ represents a transfer vector from $X_{i}$ to $X_{j}$ and when divided by Dis $s_{i, j}$, a unit vector is generated from $X_{i}$ towards $X_{j}$. Random function generates a random number which causes $\mathrm{AF} i$ to move towards position $X_{j}$ with a random percentage of Step. Nevertheless, if $f\left(X_{i}\right)>f\left(X_{j}\right)$, we choose another position $X_{i}$ by (1) and evaluate its food density, whether forward move condition is satisfied or not. After try_number times, AF does not succeed in satisfying forward condition. The AF performs free move behavior and moves one Step in searching space randomly.

2.2.2. Swarm Behavior. One of the features of fishes as a swarm is that they always try to move along other swarm members, which causes fish swarm to scatter, and the generality of the swarm is kept. In AFSA, in order to maintain the swarm generality, AFs try to move to the center position of the searching space in each of the iterations. The central position of swarm is expressed as

$$
X_{\text {Center }}=\frac{1}{N} \sum_{i=1}^{N} X_{i}
$$

In (3), $X_{\text {Center }}$ vector represents the arithmetic average of all the AFs swarm. Suppose $N$ is the population size and $n c$ is the number of AFs in Visual field (i.e., Dis $s_{i, j}<$ Visual) around $X_{\text {Center }}$ position; if $f\left(X_{\text {Center }}\right) \geq f\left(X_{i}\right)$ and $\delta>n c / N$, center position has better food density than current position and the population crowd degree in its neighborhood is not crowded, so AF $i$ moves towards central position:

$$
\begin{aligned}
X_{i+1}(t+1)= & X_{i}(t)+\frac{X_{\text {Center }}-X_{i}(t)}{\operatorname{Dis}_{i, \text { Center }}} \\
& \times[\text { Visual } \times \text { Rand }(0,1)] .
\end{aligned}
$$

If $n c=0$ or the movement condition towards central position is not satisfied, prey behavior is performed by AF $i$ alternatively.

2.2.3. Following Behavior. During the AFs moving process, when the fishes find food, neighbor fishes follow them to search for food. When the current AF in position $X_{i}$ checks its neighbor $X_{k}$, if $k n$ is the number of AFs in Visual field (i.e., Dis $_{i, j}<$ Visual) around $X_{k}$, and if $f\left(X_{k}\right) \geq f\left(X_{i}\right)$ and $\delta>k n / N$ (i.e., $\mathrm{AF} i$ in position $X_{k}$ has better food density than current position and the population density around its neighborhood is not crowded), AF $i$ moves one Step towards $X_{k}$ by the following equation:

$$
\begin{aligned}
X_{i}(t+1)= & X_{i}(t)+\frac{X_{k}-X_{i}(t)}{\operatorname{Dis}_{i, k}} \\
& \times[\text { Visual } \times \text { Rand }(0,1)] .
\end{aligned}
$$

If $\mathrm{AF} i$ has no neighbors or none of its neighbors satisfy the following conditions, prey behavior would be performed by AF $i$ optionally.

2.2.4. Free Move Behavior. AFs would move freely when they are not successful in finding food. In AFSA, the AFs would move a random Step in search space in this no-more-food condition, so it could be represented as

$$
X_{i}(t+1)=X_{i}(t)+\text { Step } \times \text { Rand }(-1,1) .
$$

The free move behavior is necessary to maintain the diversity of AFs swarm when the AFs search for a better position in problem space.

2.3. Demerits of SAFSA in Accelerometer Error Coefficients Identification. According to the characteristics of SAFSA, there are some demerits for its application to the recalibration of accelerometer error parameters [18-20]. The first one is that the AFs are lacking the application of previous experiences, which would lead the AFs to falling into local extreme during optimization process. Consequently, the SAFSAidentified accelerometer error parameters are not the optimal values. The second one is that the SAFSA lacks the balance between exploration and exploitation during optimization process, which deteriorates the convergence rate and accuracy of the AFs. Hence, it decelerates the accelerometer error coefficients optimization process and reduces the optimal precision. The last demerit is that the computational cost of SAFSA is high, which will cost more memory loads during optimization process. So it is unsuitable for accelerometerbased SINS with high real-time computational requirements. Therefore, these disadvantages should be eliminated before the AFSA is applied to the accelerometer error coefficients identification.

\section{Optimal Artificial Fish Swarm Algorithm (OAFSA)}

According to the AFSA for accelerometer error coefficients identification, the mentioned drawbacks in Section 2.3 should be avoided. At present, the varied Visual and Step parameters are used to improve the algorithm's precision and convergence rate [29]. Moreover, the secondary initialization method is also utilized for higher precision accelerometer error parameters identification $[18,20]$.

3.1. Parameters Variation on AFSA. The SAFSA has fixed value on both Visual and Step parameters, which restrict AFSA's precision and convergence rate in accelerometer error parameters identification greatly. When the initialization 
value of parameters Visual and Step is relatively large, the exploration ability of AFSA is enhanced while the exploitation ability is weakened. Conversely, if the Visual and Step parameters are relatively small, AFSA's exploration ability is weakened and the exploitation ability of the algorithm is enhanced during the optimization process [18-20]. Therefore, varied Visual and Step parameters are adopted:

$$
\begin{aligned}
\text { Visual } & =\text { Visual } \lambda+\text { Visual }_{\min }, \\
\text { Step } & =\text { Step } \cdot \lambda+\text { Step }_{\min }, \\
\lambda & =\exp \left(-3 \times\left(\frac{G}{G_{\max }}\right)\right),
\end{aligned}
$$

where $G$ and $G_{\max }$ denote the current iteration times and the maximum iteration times during the optimization process. And $\lambda$ denotes the attenuation function, which could balance the exploration and exploitation abilities during the overall optimization process. At the beginning stage of the optimization process, the Visual and Step parameters are relatively large. So the AFs exploration ability is greater than the exploitation ability and the AFs could find a better position in a relatively large space, while, with the increase of the iterations, the $\lambda$ parameter is less than ever; both Visual and Step parameters become less with the increase of iterations. Therefore, the AFs exploration ability is weakened and exploitation ability is enhanced, and the AFs could implement higher precision with more local optimization time.

3.2. The Secondary Initialization Method. After dozens of iterations, the objective or indicator function starts to present divergence tendency because the AFs fall into the local extreme by unsuccessful prey behavior [26]. The former AFs parameters and the optimized accelerometer error parameters have reached their limits to implement higher precision during optimization process. In this case, the secondary initialization method is utilized to the AFs swarm and the related accelerometer error parameters identification procedures [18-20]. Firstly, observe the variation tendency of indicator function until it presents divergence tendency, which is caused by unsuccessful prey behavior. And then, store all the AFs parameters and the corresponding accelerometer error coefficients when the indicator function reaches the lowest point. After that, reload the changed AFs parameters and former saved accelerometer error coefficients manually, which were obtained from the former optimization process. Finally, execute the AFSA optimization process again and let the optimal indicator function reach higher optimization precision. However, in secondary initialization method, the selection of the lowest indicator function point at first stage is artificially aided. The reload process of AFs parameters and accelerometer error coefficients are also completed manually. This means the method is nonautonomous during optimization process.

3.3. Demerits of OAFSA in Accelerometer Error Coefficients Identification. When OAFSA is used for accelerometer error parameters identification, only the second drawback of SAFSA is eliminated, but the other two drawbacks are not avoided during the optimization process. Moreover, by inducing the secondary initialization method, the lowest indicator function point selection at the first stage is artificially aided. The reload process of AF parameters and accelerometer error coefficients are completed manually. This means the method is nonautonomous during the optimization process. Therefore, there are also some shortcomings when OAFSA is applied to accelerometer error parameters identification.

\section{Novel Artificial Fish Swarm Algorithm (NAFSA)}

4.1. Parameters of NAFSA. In order to solve the abovementioned demerits of SAFSA and the OAFSA when they are applied in accelerometer error coefficients identification, a novel artificial fish swarm algorithm is proposed. At first, the Contraction Factor (CF) parameter is adopted to substitute the parameters of Step and crowd factor. Moreover, the simplification on fish swarm behaviors is also elucidated, and prey and free move behaviors are combined with some changes in a specific behavior, which is called individual behavior. And also there is another behavior called group behavior, which substitutes the following and swarm behaviors.

Suppose that there are $N$ artificial fishes in $D$-dimensional space, and the position of AF $i$ could be expressed as $X_{i}=\left(x_{i, 1}, x_{i, 2}, \ldots, x_{i, D}\right)$. AFs' Visual could be denoted as vector $\left(v_{1}, v_{2}, \ldots, v_{D}\right)$, and the dimension of every Visual vector is determined by the inner coverage of searching space dimension. Therefore, the NAFSA could use different Visual in various space ranges. The components of vector Visual are divided into many parts which make the AFs perform better global optimization ability. So the NAFSA has higher precision in global extreme optimal ranges. The CF parameter is introduced to NAFSA for choosing different Visual values in different optimal phases, which is an integer less than 1 , whether a constant or a function. Previously, the inertial weight parameter was presented in particle swarm optimization (PSO) for balancing the exploration and exploitation ability during optimization process [30]. The CF in NAFSA has the similar function to inertial weight in PSO. Here, we use random function to generate the CF in all iteration process, which is expressed as

$$
\mathrm{CF}=\mathrm{CF}_{\min }+\left(\mathrm{CF}_{\max }-\mathrm{CF}_{\min }\right) \times \operatorname{Rand}(0,1) .
$$

The above equation generates a random $\mathrm{CF}$ in range $\left[\mathrm{CF}_{\min }, \mathrm{CF}_{\max }\right]$. Therefore, the ith element of vector Visual in each iteration process could be expressed as

$$
\operatorname{Visual}_{i}(t+1)=\operatorname{Visual}_{i}(t) \times \mathrm{CF} .
$$

Next, the NAFSA behaviors will be discussed.

\subsection{Behaviors of NAFSA}

4.2.1. Individual Behavior. The individual behavior is made up of prey and free move behaviors. The AF $i$ in position $X_{i}(t)$ 
tries several times of movement to a better position. In each iteration process, AF $i$ will occupy the position $X_{j}(t)$ by (1) and then evaluate its food density. If $f\left(X_{i}\right) \geq f\left(X_{j}\right)$, then the next position of AF $i$ is represented as

$$
X_{i}(t+1)=X_{j}
$$

Because the position $X_{j}(t)$ is within the Visual range of AF $i$, the movement distance of AF $i$ would be less than or equal to Visual vector in the same dimension. If $f\left(X_{i}\right) \geq$ $f\left(X_{j}\right)$, the AF $i$ will move to better positions with several times' iteration by (10) or (1) and (10). However, if $f\left(X_{i}\right)<$ $f\left(X_{j}\right)$ is satisfied, the AF would not move towards $X_{j}(t)$ and it will find a better position from its previous position. Therefore, when performing single individual behavior, the AF could find a better position by trying several times. Otherwise, if AF $i$ could not find a better position after all attempts, the AF could move a Step randomly within its Visual range:

$$
X_{i}(t+1)=X_{i}(t)+\operatorname{Visual} \times \operatorname{Rand}(-1,1) .
$$

In NAFSA, each AF moves towards a better position by individual behavior. But when it failed, it will perform random behavior within its Visual range and may discard its previous position, which may find a worse position in searching space. Nevertheless, in order to keep the AF swarm diversity and find a better position in later optimal behavior, performing the random behavior is necessary for the AF. Moreover, the AF position searched by random behavior would not be used in the best AF position, so the best AF position would not be lost even if the AF could not find better position. In this case, the best AF position is what has been searched for previously. Therefore, in NAFSA, the current AF position is the best position, so the bulletin parameter is no longer a necessity.

4.2.2. Group Behavior. To keep the swarm characteristics of all the fishes and make the moving of AF to the best position are the two main targets in group behavior. The center position of AF swarm is obtained by (3). If $f\left(X_{\text {Center }}\right)>$ $f\left(X_{i}\right)$ is satisfied, then the next position of AF $i$ is

$$
\begin{aligned}
X_{i}(t+1)= & X_{i}(t)+\frac{X_{\text {Center }}-X_{i}(t)}{\operatorname{Dis}_{i, \text { Center }}} \\
& \times[\text { Visual } \times \text { Rand }(0,1)] .
\end{aligned}
$$

Visual in (12) is a vector, and the random function generates a $D$-dimensional random number. If $f\left(X_{\text {Center }}\right) \leq$ $f\left(X_{i}\right)$, the AF $i$ could not move towards center position, while it could move towards the best position in searching space:

$$
\begin{aligned}
X_{i}(t+1)= & X_{i}(t)+\frac{X_{\text {Best }}-X_{i}(t)}{\operatorname{Dis}_{i, \text { Best }}} \\
& \times[\text { Visual } \times \text { Rand }(0,1)] .
\end{aligned}
$$

Therefore, the AFs in a worse position would move towards center position by comparing with center position.
When the position is better than center position, it will move towards best AF swarm position. Therefore, all the AFs will reach the best position by performing a group behavior. Consequently, in NAFSA, the best position searched by fish swarm would be adopted to accelerate the convergence rate with all AFs' movement. So the group behavior is used to maintain the fishes swarm characteristics and avoid reducing in swarm diversity.

In group behavior, the center position AF may have better food density (indicator function) than best AF position. AF moves towards center position by (12), but worse position may exist between current position and center position. Then the AF position may get worse or even lose its best position by executing (12). Therefore, if the indicator function of center position is better than best AF position, the best AF position is determined by the following equation:

$$
X_{\text {Best }}(t+1)=X_{\text {Center }} \text {. }
$$

The above equation is executed only when $f\left(X_{\text {Center }}\right)<$ $f\left(X_{\text {Best }}\right)$, while the other AFs' movement by using (12) helps to maintain the diversity of the fish swarm.

\section{Triaxial Accelerometer Error Coefficients Identification by NAFSA}

In this section, the triaxial accelerometer static error model will be demonstrated at first. And then the optimization indicator function derivation process is given in detail. Finally, the triaxial accelerometer error coefficients identification procedures with NAFSA will be provided specifically.

5.1. Static Error Model of Triaxial Accelerometer. The purpose of the error coefficients identification is to identify accelerometer error parameters quickly and accurately. There are various identification methodologies for triaxial accelerometer error parameters [31-35]. The static error model for triaxial accelerometer is shown as follows [35]:

$$
\begin{aligned}
{\left[\begin{array}{c}
\frac{N_{a x}}{K_{a x}} \\
\frac{N_{a y}}{K_{a y}} \\
\frac{N_{a z}}{K_{a z}}
\end{array}\right]=} & {\left[\begin{array}{ccc}
1 & E_{a x z} & E_{a x y} \\
E_{a y z} & 1 & E_{a y x} \\
E_{a z y} & E_{a z x} & 1
\end{array}\right]\left[\begin{array}{c}
A_{x} \\
A_{y} \\
A_{z}
\end{array}\right]+\left[\begin{array}{c}
A_{0 x} \\
A_{0 y} \\
A_{0 z}
\end{array}\right] } \\
& +\left[\begin{array}{c}
\frac{K_{2 x} A_{x}^{2}}{K_{a x}} \\
\frac{K_{2 y} A_{y}^{2}}{K_{a y}} \\
\frac{K_{2 z} A_{z}^{2}}{K_{a z}}
\end{array}\right],
\end{aligned}
$$

where $K_{a i}(i=x, y, z)$ denote the triaxial accelerometer scale factors, $E_{a i j}(i, j=x, y, z ; i \neq j)$ denote the triaxial accelerometer axis misalignment errors during installation, $N_{g i}(i=x, y, z)$ denote the triaxial accelerometers output data, $A_{i}(i=x, y, z)$ denote the turntable alignment axis 
input linear accelerations, $K_{2 i}(i=x, y, z)$ denote the quadratic nonlinear coefficients of triaxial accelerometer, and $A_{o i}(i=x, y, z)$ denote the triaxial accelerometer biases. Therefore, there are totally 15 static error parameters for triaxial accelerometer to be identified by NAFSA.

\subsection{Determining of the Optimization Indicator. The NAFSA} is terminated in one of the three conditions. The first is when the preset maximum iteration times are reached. The second condition is when the optimization indicator function during optimization process is below predefined threshold. The third condition is that when performing the next iteration, the deviation of current iteration result and the next iteration result is within an acceptable range. Therefore, the optimization indicator is a key factor for the termination condition of NAFSA optimization process. The following part will present an optimization indicator function for triaxial accelerometer error parameters identification based on NAFSA.

Theoretically, when the static triaxial accelerometer is at arbitrary space position, the accelerometer measured linear accelerations would satisfy the following equation:

$$
\sum_{i=x, y, z} A_{i}^{2}=g^{2}
$$

In (16), $A_{i}(i=x, y, z)$ are the theoretical input linear accelerations sensed by triaxial accelerometer in each axis and $g$ denotes the Earth gravity vector, which is related to the position on Earth surface.

Actually, because of the errors caused by accelerometer itself, the calculated linear accelerations are different from theoretical values. Therefore, the linear acceleration mode square error (MSE) is adopted to represent the deviation, which is derived from (16), and it is expressed as

$$
\delta g=\sum_{i=x, y, z} \widehat{A}_{i}^{2}-g^{2}
$$

where $\widehat{A}_{i}(i=x, y, z)$ denote the accelerations calculated from (15) with the stored triaxial accelerometer output data $N_{a i}(i=x, y, z)$.

Our target by using the NAFSA is to identify the error coefficients of accelerometer precisely and make the linear acceleration MSE as stable as possible. So the standard deviation function is used to evaluate the discrete degree of the accelerometer error coefficients:

$$
\sigma=\sqrt{\frac{\sum_{j=1}^{m}\left(\sum_{i=x, y, z} \widehat{A}_{i}^{2}-g^{2}\right)}{m-1} .}
$$

In (18), $m$ denotes the number of positions during optimization process.

5.3. MCS-NAFSA Implementation Procedures. In this subsection, the implementation procedures of accelerometer error coefficients identification will be explained. Two main steps are conducted to illustrate the NAFSA optimization process. At the beginning, the variation characteristics of the 15 error coefficients in triaxial accelerometer are discussed and a clustering process is described on different triaxial accelerometer error parameters. After that, the specific MCSNAFSA accelerometer error coefficients identification procedures are presented step by step.

5.3.1. Accelerometer Error Coefficients Clustering. In 15 triaxial accelerometer error coefficients' identification, different error coefficients have different influences on the linear acceleration MSE and also the NAFSA requires all the AFs to have similar characteristics during optimization process. Therefore, the clustering on accelerometer error coefficients is a necessity before the accelerometer error coefficients identified by NAFSA.

Thinking about the different error coefficients' influences on linear acceleration MSE and based on our previous experiences, the accelerometer scale factors have the highest impacts on linear acceleration MSE, followed by the biases, and then the accelerometer axis misalignment errors, and the last parameters are accelerometer quadratic nonlinear coefficients. So accelerometer error coefficients are divided into four different categories. They are 3 accelerometer scale factors $K_{a i}(i=x, y, z)$ as category one, 3 accelerometer biases $A_{o i}(i=x, y, z)$ as category two, 6 accelerometer misalignment errors $E_{a i j}(i, j=x, y, z ; i \neq j)$ as category three, and 3 accelerometer quadratic nonlinear coefficients $K_{2 i}(i=x, y, z)$ as category four. Therefore, when adopting MCS-NAFSA to identify the accelerometer error parameters, there are four main steps of optimization process that should be conducted to implement the highest precision.

5.3.2. MCS-NAFSA Accelerometer Procedures. Through the discussion in Section 5.3.1, within NAFSA accelerometer procedures, the accelerometer error coefficients identified by NAFSA is mutually independent in different categories. Hence, pseudocode of four phases of optimization process is shown in Algorithm 1, accelerometer NAFSA.

In the first phase, accelerometer scale factors $K_{a i}(i=$ $x, y, z)$ identification is optimized by NAFSA. Firstly, the AFs parameters, category two parameters $A_{o i}(i=x, y, z)$, category three parameters $E_{a i j}(i, j=x, y, z ; i \neq j)$, and category four parameters $K_{2 i}(i=x, y, z)$ are all initialized. After that, each AF $i$ performs individual behavior and moves to a better position based on the optimization outcome. Subsequently, each AF $i$ executes group behavior with respect to its new position. Finally, repeat this process for $N$ times, and we could obtain the mean value $\overline{\widehat{K}}_{a i}(i=x, y, z)$ as the accelerometer scale factors.

In the second phase, accelerometer biases $A_{o i}(i=x, y, z)$ identification is presented by NAFSA. At the beginning, the AFs parameters, category three parameters $E_{a i j}(i, j=$ $x, y, z ; i \neq j)$, category four parameters $K_{2 i}(i=x, y, z)$, and accelerometer scale factors $\widehat{K}_{a i}(i=x, y, z)$ are all loaded. Next, all the AFs execute individual behavior and group behavior, respectively. At last, repeat this process for $N$ times, and we could obtain the mean value $\widehat{\widehat{A}}_{o i}(i=x, y, z)$ as the accelerometer biases.

In the third phase, accelerometer axis misalignment errors $E_{a i j}(i, j=x, y, z ; i \neq j)$ identification is demonstrated 


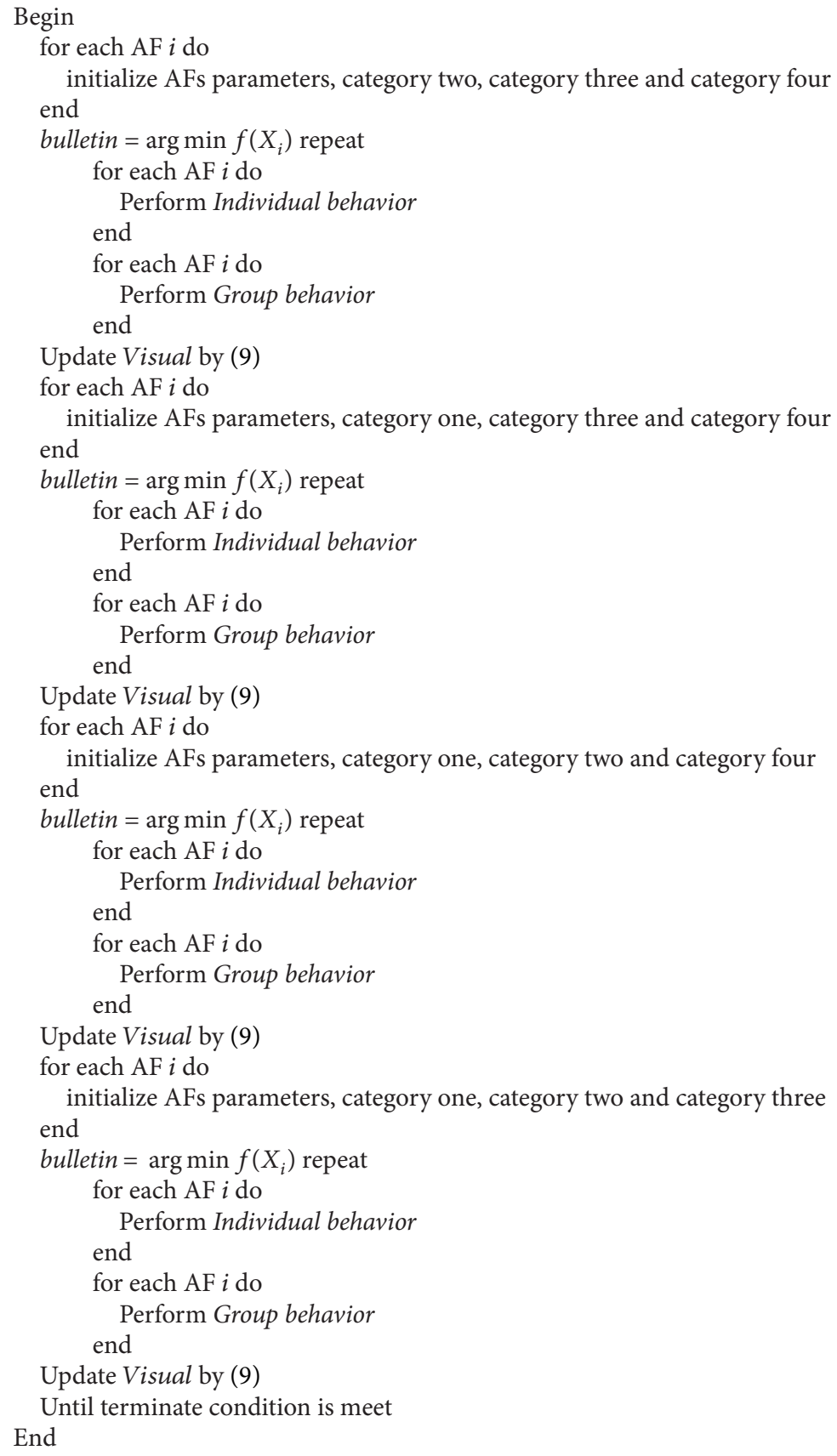

Algorithm 1: Accelerometer NAFSA.

by NAFSA. At first, the AFs parameters, category four parameters $K_{2 i}(i=x, y, z)$, accelerometer scale factors $\overline{\widehat{K}}_{a i}(i=x, y, z)$, and accelerometer biases $\overline{\widehat{A}}_{o i}(i=x, y, z)$ are all loaded in initialization process. Second, all the AFs execute individual behavior and groupbehavior, respectively. And last, repeat this process for $N$ times, and we could obtain the mean value $\widehat{\widehat{E}}_{a i j}(i, j=x, y, z ; i \neq j)$ as the accelerometer axis misalignment errors.

In the last phase, accelerometer quadratic nonlinear coefficients $K_{2 i}(i=x, y, z)$ identification is processed by NAFSA.
At the outset, the AFs parameters, accelerometer scale factors $\overline{\widehat{K}}_{a i}(i=x, y, z)$, accelerometer axis misalignment errors $\overline{\widehat{E}}_{a i j}(i, j=x, y, z ; i \neq j)$, and accelerometer biases $\overline{\widehat{A}}_{o i}(i=$ $x, y, z)$ are all loaded in initialization process. Then, all the AFs execute individual behavior and group behavior, respectively. And last, repeat this process for $N$ times, and we could obtain the mean value $\overline{\widehat{K}}_{2 i}(i=x, y, z)$ as the accelerometer quadratic nonlinear coefficients.

Finally, repeat the above four phases until the accelerometer error coefficients meet the termination conditions, when 
TABLE 1: NAFSA triaxial accelerometer preset AFs parameters.

\begin{tabular}{|c|c|c|c|c|}
\hline \multirow[b]{2}{*}{ Accelerometer parameter types } & \multicolumn{4}{|c|}{ NAFA AFs parameters } \\
\hline & Visual $_{d}$ & AFs numbers $N$ & $\begin{array}{l}\text { Maximum iteration times } \\
\qquad G_{\max }\end{array}$ & $\begin{array}{c}\text { Contraction factors } \\
{\left[\mathrm{CF}_{\min }, \mathrm{CF}_{\max }\right]}\end{array}$ \\
\hline$K_{a i}$ & $(5.0000,2.0000,0.1000)$ & 50 & 60 & [0.000001, 0.999999] \\
\hline$A_{0 i}$ & $(0.0050,0.0020,0.0001)$ & 50 & 60 & [0.000001, 0.999999] \\
\hline$E_{a i j}$ & $\begin{array}{c}(0.0005,0.0002,0.00001 \\
0.000005,0.000002 \\
0.000001)\end{array}$ & 50 & 60 & [0.000001, 0.999999] \\
\hline$K_{2 i}$ & $(0.0050,0.0020,0.0001)$ & 50 & 60 & [0.000001, 0.999999] \\
\hline
\end{tabular}

TABLE 2: Triaxial accelerometer error coefficients preset values.

\begin{tabular}{lccc}
\hline $\begin{array}{l}\text { Parameters } \\
\text { types }\end{array}$ & Preset parameters \\
\hline $\begin{array}{l}K_{a i}(i=x, y, z) \\
(\text { pulse/g) }\end{array}$ & {$\left[\begin{array}{llll}701.887665 & 701.777454 & 701.663265\end{array}\right]$} \\
\hline $\begin{array}{l}A_{0 i}(i=x, y, z) \\
(\% / h)\end{array}$ & {$\left[\begin{array}{llll}-0.006662897 & 0.012276399 & 0.013897022\end{array}\right]$} \\
\hline $\begin{array}{l}1 \\
E_{a i j}(i, j= \\
x, y, z ; i \neq j)\left(^{\circ}\right)\end{array}$ & {$\left[\begin{array}{cccc}-0.00057700 & 1 & -0.00033773 \\
0.00022593 & -0.00034751 & 1\end{array}\right]$} \\
\hline$K_{2 i}(i=x, y, z)$ & {$\left[\begin{array}{cccc}0.0001134 & 0.0001765 & 0.000 & 1762\end{array}\right]$}
\end{tabular}

optimization indicator reaches $\sigma<10^{-8}$ or the iteration times to a certain preset number.

\section{Simulation Experiment and Discussion}

In this section, the triaxial accelerometer error coefficients simulation experiment is conducted by MCS-NAFSA. Before the simulation experiment, the AFs parameters and the nonoptimized triaxial accelerometer error parameters at each phase should be preset. Subsequently, the simulation process on the triaxial accelerometer error parameters is shown by MCS-NAFSA method.

6.1. Simulation Parameters Preset. Section 4.1 described all the AFs parameters during optimization process, and all the AFs preset parameters of triaxial accelerometer before the accelerometer error parameters identified are listed in Table 1.

Meanwhile, in Section 5.3.2, when one-category triaxial accelerometer parameters are identified by NAFSA, the other three categories parameters also have influences on linear acceleration MSE. Therefore, the triaxial accelerometer error coefficients preset values are shown in Table 2.

It is worth noting that, in each iteration, the dimensions of vector Visual $_{d}$ listed in Table 1 are equal to the number of triaxial accelerometer error parameters. So the vector dimensions of Visual $_{d}$ on triaxial accelerometer axis misalignment errors are different from triaxial accelerometer scale factors, biases, and quadratic nonlinear coefficients. CF is a positive number less than 1 whose minimum value and maximum value are preset as 0.000001 and 0.999999, respectively.
In Table 2, in order to reduce the influences of other three categories' triaxial accelerometer error parameters on linear acceleration MSE during one-category triaxial accelerometer error parameters optimization process, the preset values of triaxial accelerometer error parameters are based on the conventional 24-position calibration method [32, 35], which is aided by the high-precision turntable in indoor environments.

6.2. Simulation Results and Discussion. After all the parameters preset procedure (i.e., the initialization process) is completed, the AFs start to execute the NAFSA optimization procedure. In order to increase the triaxial accelerometer error parameters' credibility degree during NAFSA optimization process, 100-time MCS is conducted after the single NAFSA to bring in the random factors. Figure 1 shows the triaxial accelerometer scale factors NAFSA identification results with 100 -time MCS. Meanwhile, Figure 2 demonstrates the triaxial accelerometer biases NAFSA identification results with 100 -time MCS. Figure 3 presents the triaxial accelerometer axis misalignment errors NAFSA identification results with 100-time MCS. Figure 4 displays the triaxial accelerometer quadratic nonlinear coefficients NAFSA identification results with 100-time MCS.

From Figures 1 to 4 , all triaxial accelerometer error parameters fluctuate with the MCS process within a relatively small range and the results reveal that the triaxial accelerometer error parameters are influenced by triaxial accelerometer usage environment and the inherent physical properties of the instrument. However, the precision of simulation results is also likely to be deteriorated by random factors, such as the algorithmic error and computer's error, which are usually being neglected and unable to be eliminated during single NAFSA optimization. So in this phase, to reduce the effects of these random factors, the data smoothing method is also utilized to obtain the mean value of the MCS-NAFSA simulation results on accelerometer error coefficients identification. All the simulation results by smoothing method and the corresponding statistical properties are listed in Table 3.

Through the comparison of preset parameters and the NAFSA identification results in Table 3, we can summarize that the relative errors amplitude of each parameter is substantially small. The triaxial accelerometer scale factors $K_{a i}(i=x, y, z)$ relative error magnitude is small enough to $10^{-2} \sim 10^{-3} \mathrm{ppm}$, which could fully meet the high-precision 

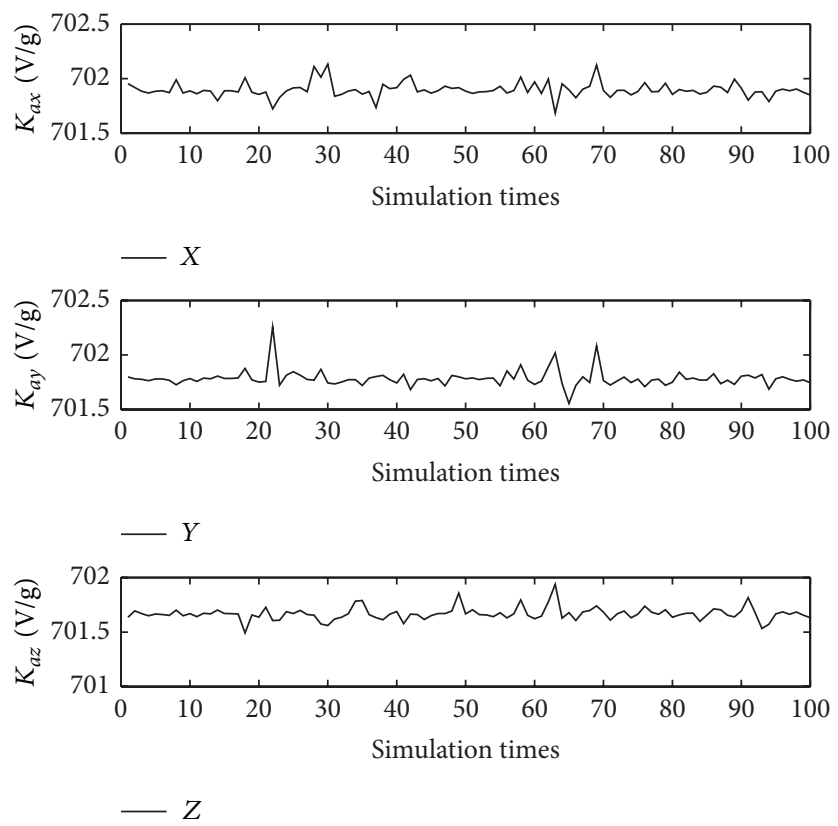

FIGURE 1: Triaxial accelerometer scale factors: MCS-NAFSA curves.
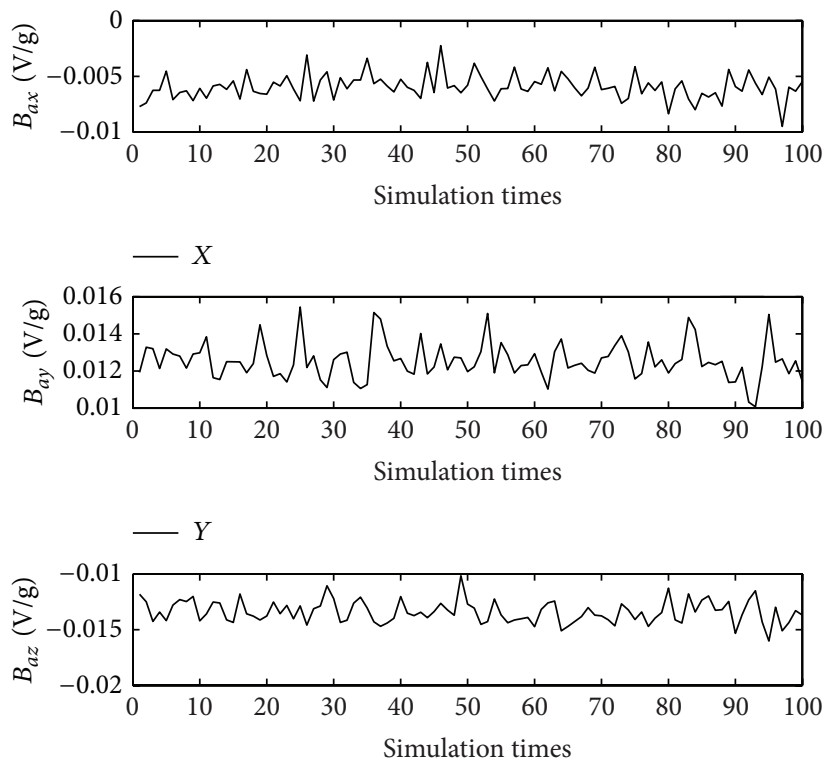

$-Z$

FIgURE 2: Triaxial accelerometer biases: MCS-NAFSA curves.

navigation requirements. Meanwhile, the relative errors of triaxial accelerometer biases $A_{o i}(i=x, y, z)$ and axis misalignment errors $E_{a i j}(i, j=x, y, z ; i \neq j)$ could reach $10^{-3} \sim$ $10^{-4}$ in magnitude, which is completely enough to satisfy the accelerometer-based SINS precision demands. Moreover, the relative error of triaxial accelerometer quadratic nonlinear coefficients $K_{2 i}(i=x, y, z)$ is within $10^{-2} \sim 10^{-3}$ in magnitude and also could meet the precision requirements of the accelerometer-based SINS. For comparison, in OAFSA, the triaxial accelerometer scale factors relative error magnitude is $10^{-1} \sim 10^{-2} \mathrm{ppm}$ and the triaxial accelerometer biases and axis misalignment errors relative error magnitudes are $10^{-2} \sim 10^{-3}$, and the relative error of the triaxial accelerometer quadratic nonlinear coefficientsis also within $10^{-2} \sim 10^{-3}$ in magnitude [18-20]. Therefore, theoretically, the precision of the proposed NAFSA identification results is an order of magnitude higher than OAFSA in triaxial accelerometer error parameters identification. Therefore, the proposed NAFSA not only satisfies the precision requirements of the highprecision accelerometer-based SINS, but also presents lower 

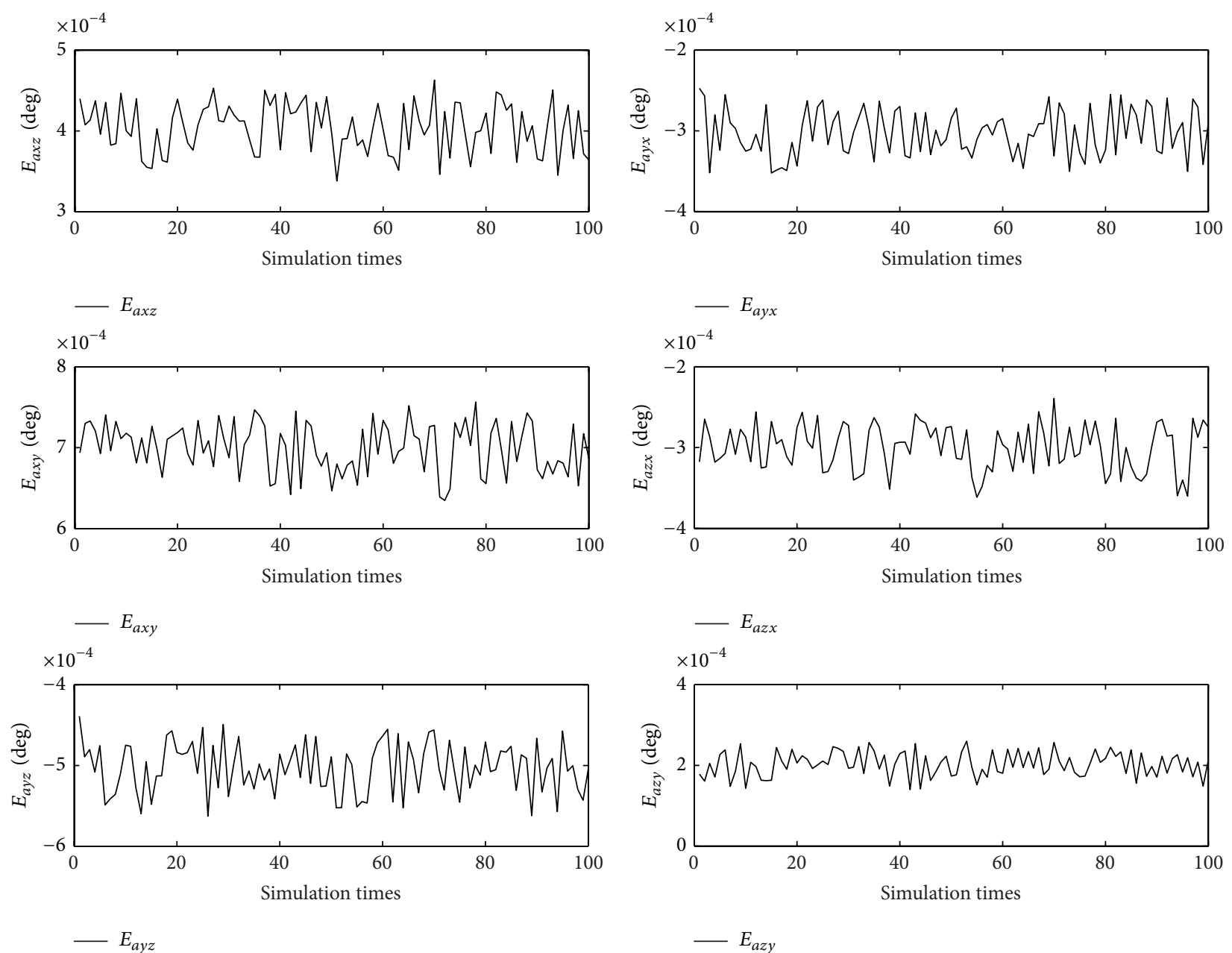

FIgURE 3: Triaxial accelerometer axis misalignment errors: MCS-NAFSA curves.

TABLE 3: Triaxial accelerometer error parameters MCS-NAFSA identification results.

\begin{tabular}{lcccc}
\hline Parameters & Preset value & MCS-NAFSA identification result & Relative error & Standard deviation \\
\hline$K_{a x}(\mathrm{volt} / \mathrm{g})$ & 701.887665 & 701.887542 & $1.752417 e-7$ & 0.06787 \\
$K_{a y}(\mathrm{volt} / \mathrm{g})$ & 701.777454 & 701.777946 & $7.010765 e-7$ & 0.07613 \\
$K_{a z}(\mathrm{volt} / \mathrm{g})$ & 701.663265 & 701.663155 & $1.567704 e-7$ & 0.05935 \\
$A_{o x}(\mathrm{volt} / \mathrm{g})$ & -0.006662897 & -0.006669367 & $9.701071 e-4$ & 0.001124 \\
$A_{o y}(\mathrm{volt} / \mathrm{g})$ & 0.012276399 & 0.012272449 & $3.218591 e-4$ & 0.000995 \\
$A_{o z}(\mathrm{volt} / \mathrm{g})$ & 0.013897022 & 0.013893412 & $2.598354 e-4$ & 0.001011 \\
$E_{a x z}(\mathrm{deg})$ & 0.00040520 & 0.00040370 & 0.00371563 & $3.091 e-5$ \\
$E_{a x y}(\mathrm{deg})$ & 0.00077257 & 0.00077564 & 0.00395802 & $3.058 e-5$ \\
$E_{a y z}(\mathrm{deg})$ & -0.00057700 & -0.00057029 & 0.01176594 & $3.127 e-5$ \\
$E_{a y x}(\mathrm{deg})$ & -0.00033773 & -0.00033027 & 0.02258758 & $2.892 e-5$ \\
$E_{a z y}(\mathrm{deg})$ & 0.00022593 & 0.00022029 & 0.02560261 & $3.093 e-5$ \\
$E_{a z x}(\mathrm{deg})$ & -0.00034751 & -0.00034299 & 0.01317823 & $2.869 e-5$ \\
$K_{2 x}\left(\mathrm{volt} / \mathrm{g}^{2}\right)$ & 0.0001134 & 0.0001105 & 0.02624434 & $9.393 e-5$ \\
$K_{2 y}\left(\mathrm{volt} / \mathrm{g}^{2}\right)$ & 0.0001765 & 0.0001706 & 0.03458382 & $9.393 e-5$ \\
$K_{2 z}\left(\mathrm{volt} / \mathrm{g}^{2}\right)$ & 0.0001762 & 0.0001728 & 0.01967593 & $9.393 e-5$ \\
\hline
\end{tabular}



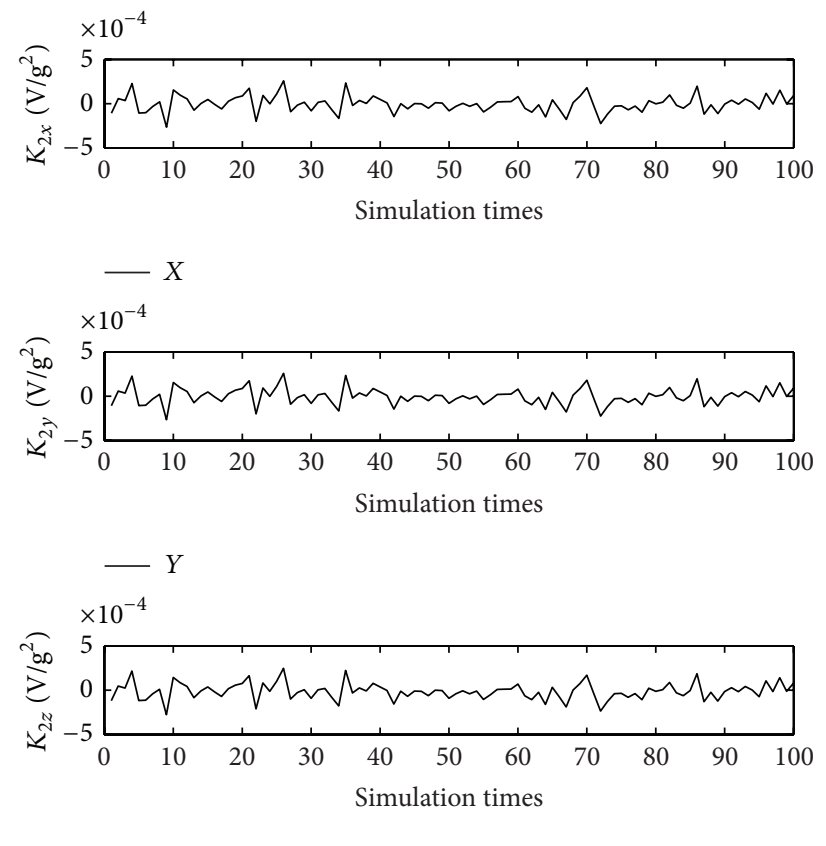

$-Z$

FIgURE 4: Triaxial accelerometer quadratic nonlinear coefficients: MCS-NAFSA curves.

relative errors than previous OAFSA in all triaxial error coefficients identification results.

Furthermore, the standard deviation of the estimates in the last column of Table 3 is an indicator for representing the stability of the estimates $[36,37]$. In Table 3 , the standard deviations of the triaxial accelerometer scale factors $K_{a i}(i=x, y, z)$ are 0.06787 (volt/g), 0.07613 (volt/g), and 0.05935 (volt/g), respectively. And the estimated standard deviations for the triaxial accelerometer biases errors $A_{o i}(i=x, y, z)$ are 0.001124 (volt/g), 0.000995 (volt/g), and 0.001011 (volt/g). Meanwhile, the estimated standard deviations of the triaxial accelerometer misalignment errors $E_{a i j}(i, j=x, y, z ; i \neq j)$ are 3.091e - 5(deg), 3.058e5 (deg), 3.127e - 5 (deg), 2.892e - 5 (deg), 3.093e - 5 (deg), and $2.869 e-5$ (deg). Moreover, the estimated standard deviations for the triaxial accelerometer quadratic nonlinear errors $K_{2 i}(i=x, y, z)$ are all $9.393 e-5\left(\mathrm{volt} / \mathrm{g}^{2}\right)$. From the standard deviations of these four categories triaxial accelerometer error parameters, the standard deviations of triaxial accelerometer scale factors are greater than triaxial accelerometer biases, and the standard deviations of triaxial accelerometer biases are also greater than triaxial accelerometer axis misalignment errors. This phenomenon is corresponding to the triaxial accelerometer error coefficients clustering principle in Section 5.3.1. More importantly, the estimated triaxial accelerometer error parameters could satisfy the preset optimization indicator by (18).

At the same time, the variation tendency of indicator functions among the SAFSA, the OAFSA, and the NAFSA, when identifying the triaxial accelerometer scale factors, is demonstrated in Figure 5, respectively.

In Figure 5, the red dotted curve denotes the SAFSA indicator function variation tendency. After 20 times of

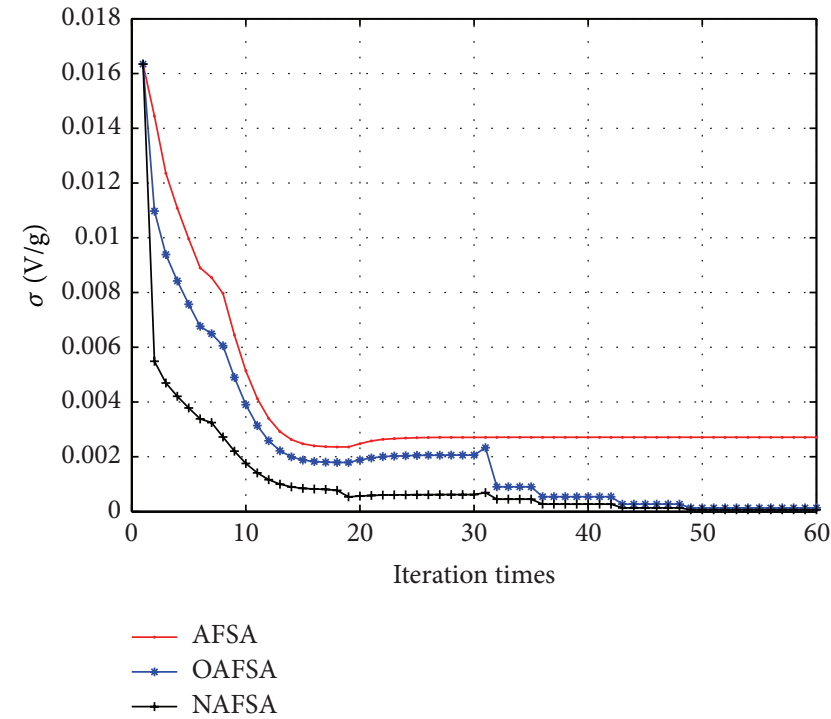

FIGURE 5: Indicator functions tendency of AFSA, OAFSA, and NAFSA.

iteration, the indicator function has a slight increase tendency and it remains stable after 24-time iteration, with the indicator function leveling at 0.002584 (volt/g). However, the OAFSA's indicator function with the blue star curve has faster convergence rate than SAFSA, but the indicator function begins divergence after 20 times of iteration. The secondary initialization method is adopted in 30 times of iteration to decrease the divergence tendency and to improve the convergence precision. For comparison, the proposed NAFSA optimization indicator function is shown with the black 


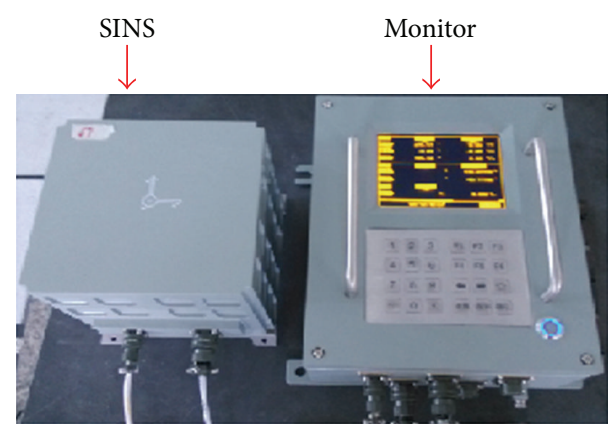

FIGURE 6: Triaxial accelerometer-based SINS.

TABLE 4: Accelerometer performance indicators.

\begin{tabular}{lc}
\hline Parameter items & Performance indicators \\
\hline Accelerometer dynamic range & $\pm 300 \mathrm{~g}$ \\
Accelerometer bias & $10^{-5} \mathrm{~g}$ \\
Accelerometer linearity & $30 \mathrm{ppm}$ \\
Accelerometer scale factor & $10 \mathrm{ppm}$ \\
\hline
\end{tabular}

plus curve in Figure 5. We can conclude that the proposed NAFSA has better convergence rate than the OAFSA and the SAFSA because of the reduction on algorithm's complexity. Moreover, it is evident that the NAFSA indicator function is always convergent during the optimization progress, which is the usage of previous experiences of AFs. Therefore, the proposed NAFSA has better performances in convergence rate and reliability of the optimized results than the SAFSA and the OAFSA.

\section{Verification Experiments and Discussion}

To validate the feasibility and priorities of the proposed NAFSA on triaxial accelerometer error parameters optimization, the static 24-position verification experiment and the accelerometer-based SINS static navigation experiment are conducted, respectively. Additionally, for comparison, the experiments with conventional calibration and Section 3 that described OAFSA on triaxial accelerometer error parameters identification are also performed.

In both experiments, the triaxial accelerometer-based SINS is developed by the Institute of Inertial Navigation and Measurement \& Control Technology at the Harbin Engineering University. The main performance indicators of triaxial accelerometer are demonstrated in Table 4. Figure 6 shows the triaxial accelerometer-based SINS in experiments.

\subsection{Triaxial Accelerometer Static 24-Position Verification} Experiment. After the triaxial accelerometer error coefficients are identified by adopting the proposed MCS-NAFSA, a static 24-position verification experiment is conducted to testify the precision of the triaxial accelerometer error parameters. Meanwhile, the triaxial accelerometer error parameters identified by the OAFSA are also testified in this experiment to reveal the advantages of the proposed MCS-NAFSA. In 24-position high-precision turntable verification experiment, when $x$-axis accelerometer points to north direction, the

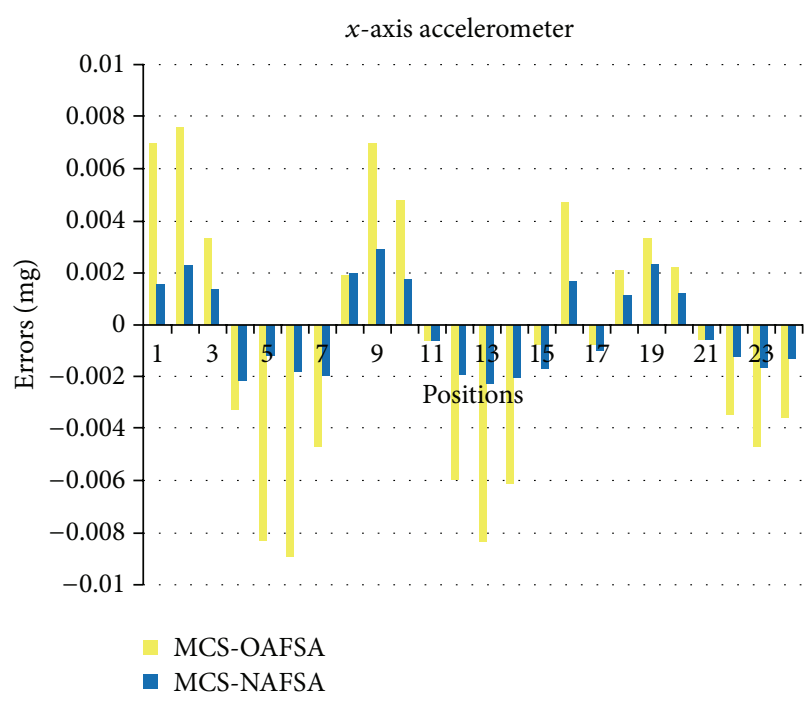

Figure 7: $x$-axis measurement errors in 24 positions.

other two axes of the accelerometer start from horizontal and vertical directions, respectively. After that, rotate the turntable axis parallel to $x$-axis accelerometer at every $45^{\circ}$ and acquire the triaxial accelerometer output data in each position. So we can obtain 8-position triaxial accelerometer output data in $360^{\circ}$. Similarly, we point the $y$ - and $z$-axis accelerometer at north direction, respectively, and rotate the turntable as $x$-axis accelerometer, and another 16 positions' accelerometer output data can also be obtained. Therefore, there are totally 24 positions utilized to testify the triaxial accelerometer measurement precision. Tables 5 and 6 list the triaxial accelerometer measurement values with the error model in Section 5.1, and the error parameters are identified by the MCS-OAFSA and the MCS-NAFSA, respectively.

In Tables 5 and 6 , the theoretical triaxial accelerometer measurement values are listed from the second column to the fourth column. Moreover, the calculated triaxial accelerometer measurement values by 24 -position verification experiment are listed from the fifth column to the seventh column. After that, the corresponding measurement errors of the triaxial accelerometer are shown from the eighth column to the tenth column. More intuitively, the comparison results of the triaxial accelerometer measurement errors in 24 positions with the error parameters identified by MCSOAFSA and MCS-NAFSA are demonstrated from Figures 7 to 9 . Consequently, the corresponding statistical results in mean values and standard deviations of these measurement errors are displayed in Table 7.

In Figure 7, the $x$-axis accelerometer measurement errors in 24 positions with the error parameters identified by MCSOAFSA vary from $-0.008930 \mathrm{mg}$ to $0.007561 \mathrm{mg}$ while the $x$-axis accelerometer measurement errors in 24 positions with the error parameters identified by MCS-NAFSA change between $-0.002243 \mathrm{mg}$ and $0.002855 \mathrm{mg}$. In Figure 8, the $y$ axis accelerometer measurement errors in 24 positions with the error parameters identified by MCS-OAFSA vary from $-0.004454 \mathrm{mg}$ to $0.007663 \mathrm{mg}$ while the $y$-axis accelerometer measurement errors in 24 positions with the error parameters 
TABLE 5: Triaxial accelerometer measurement values with MCS-OAFSA identified parameters.

\begin{tabular}{|c|c|c|c|c|c|c|c|c|c|}
\hline & \multicolumn{3}{|c|}{ Theoretical values $\left(\mathrm{m} / \mathrm{s}^{2}\right)$} & \multicolumn{3}{|c|}{ Calculated values $\left(\mathrm{m} / \mathrm{s}^{2}\right)$} & \multicolumn{3}{|c|}{ Measurement errors (mg) } \\
\hline & $A_{x}^{T}$ & $A_{y}^{T}$ & $A_{z}^{T}$ & $A_{x}^{c}$ & $A_{y}^{c}$ & $A_{z}^{c}$ & $\delta A_{x}$ & $\delta A_{y}$ & $\delta A_{z}$ \\
\hline \multirow{8}{*}{$\begin{array}{l}x \text {-axis point } \\
\text { North }\end{array}$} & 0 & 0 & 9.806550 & 0.000068 & -0.000020 & 9.806536 & 0.006964 & -0.002085 & -0.001399 \\
\hline & 0 & 6.934278 & 6.934278 & 0.000074 & 6.934267 & 6.934240 & 0.007561 & -0.001125 & -0.003839 \\
\hline & 0 & 9.80655 & 0 & 0.000033 & 9.806562 & -0.000048 & 0.003327 & 0.001231 & -0.004862 \\
\hline & 0 & 6.934278 & -6.934278 & -0.000032 & 6.934313 & -6.934316 & -0.003256 & 0.003604 & -0.003870 \\
\hline & 0 & 0 & -9.806550 & -0.000082 & 0.000045 & -9.806564 & -0.008333 & 0.004603 & -0.001444 \\
\hline & 0 & -6.934278 & -6.934278 & -0.000088 & -6.934242 & -6.934268 & -0.008930 & 0.003643 & 0.000996 \\
\hline & 0 & -9.80655 & 0 & -0.000046 & -9.806537 & 0.000020 & -0.004696 & 0.001287 & 0.002019 \\
\hline & 0 & -6.934278 & 6.934278 & 0.000019 & -6.934289 & 6.934288 & 0.001887 & -0.001085 & 0.001027 \\
\hline \multirow{8}{*}{$\begin{array}{l}y \text {-axis point } \\
\text { North }\end{array}$} & 0 & 0 & 9.806550 & 0.000068 & -0.000020 & 9.806536 & 0.006964 & -0.002085 & -0.001399 \\
\hline & -6.934278 & 0 & 6.934278 & -6.934231 & 0.000029 & 6.934249 & 0.004774 & 0.002934 & -0.002987 \\
\hline & -9.80655 & 0 & 0 & -9.806556 & 0.000068 & -0.000036 & -0.000614 & 0.006972 & -0.003659 \\
\hline & -6.934278 & 0 & -6.934278 & -6.934337 & 0.000075 & -6.934308 & -0.006043 & 0.007663 & -0.003019 \\
\hline & 0 & 0 & -9.80655 & -0.000082 & 0.0000452 & -9.8065642 & -0.008333 & 0.004603 & -0.001444 \\
\hline & 6.934278 & 0 & -6.934278 & 6.934218 & -0.000004 & -6.934277 & -0.006143 & -0.000416 & 0.000144 \\
\hline & 9.80655 & 0 & 0 & 9.806543 & -0.000044 & 0.000008 & -0.000755 & -0.004454 & 0.000815 \\
\hline & 6.934278 & 0 & 6.934278 & 6.934324 & -0.000050 & 6.934280 & 0.004674 & 0.005145 & -0.000176 \\
\hline \multirow{8}{*}{$\begin{array}{l}z \text {-axis point } \\
\text { North }\end{array}$} & 9.806550 & 0 & 0 & 9.806543 & -0.000044 & 0.000008 & -0.000755 & -0.004454 & 0.000815 \\
\hline & 6.934278 & 6.934278 & 0 & 6.934299 & 6.934251 & -0.000022 & 0.002103 & -0.002800 & -0.002273 \\
\hline & 0 & 9.80655 & 0 & 0.000033 & 9.806562 & -0.000048 & 0.003327 & 0.001230 & -0.004862 \\
\hline & -6.934278 & 6.934278 & 0 & -6.934256 & 6.934330 & -0.000053 & 0.002202 & 0.005279 & -0.005436 \\
\hline & -9.80655 & 0 & 0 & -9.806556 & 0.000068 & -0.000036 & -0.000614 & 0.006972 & -0.003659 \\
\hline & -6.934278 & -6.934278 & 0 & -6.934312 & -6.934226 & -0.000006 & -0.003472 & 0.005319 & -0.000570 \\
\hline & 0 & -9.80655 & 0 & -0.000046 & -9.806537 & 0.000020 & -0.004696 & 0.001287 & 0.002019 \\
\hline & 6.934278 & -6.934278 & 0 & 6.934243 & -6.934305 & 0.000025 & -0.003571 & -0.002761 & 0.002593 \\
\hline
\end{tabular}

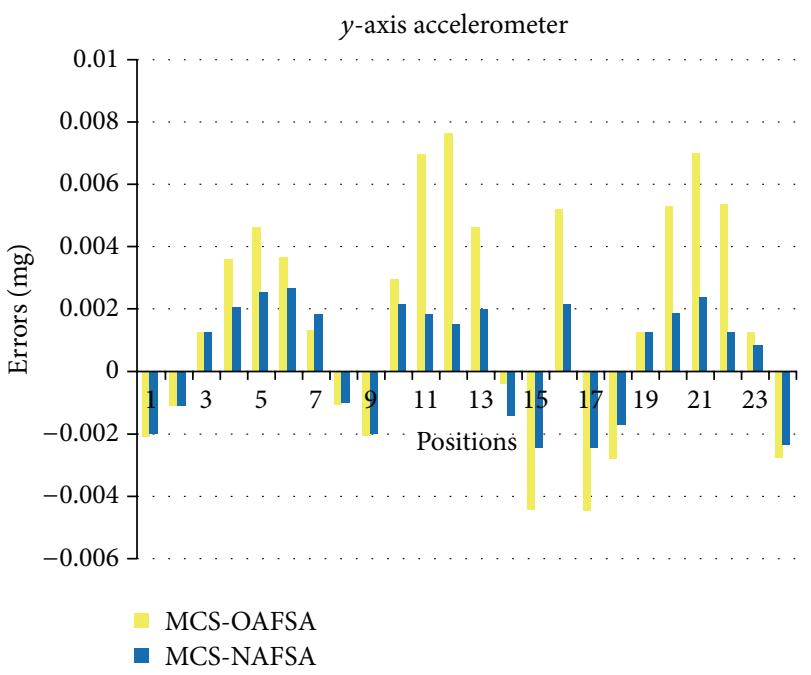

FIGURE 8: $y$-axis measurement errors in 24 positions.

identified by MCS-NAFSA change between $-0.002447 \mathrm{mg}$ and $0.002651 \mathrm{mg}$. In Figure 9, the $z$-axis accelerometer measurement errors in 24 positions with the error parameters identified by MCS-OAFSA vary from $-0.005436 \mathrm{mg}$ to

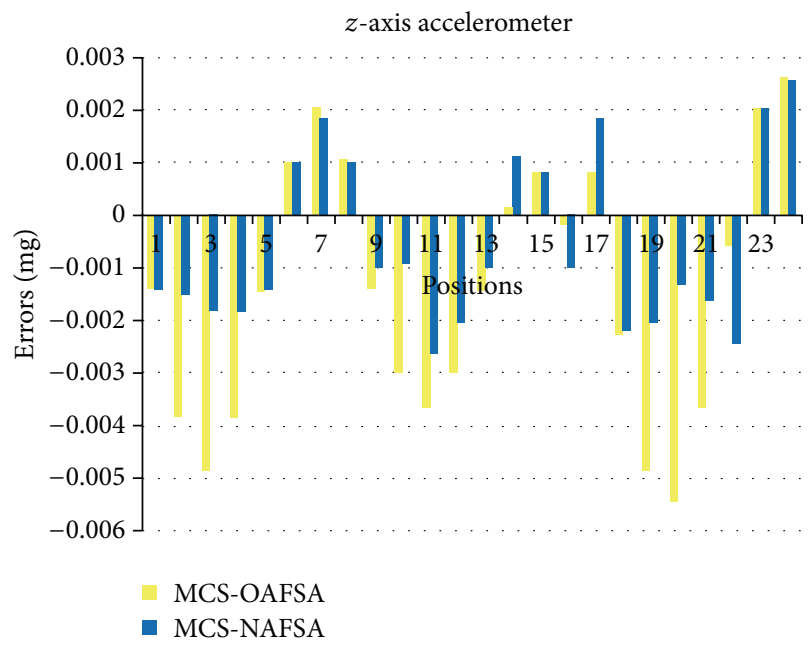

FIGURE 9: $z$-axis measurement errors in 24 positions.

$0.002593 \mathrm{mg}$ while the $z$-axis accelerometer measurement errors in 24 positions with the error parameters identified by MCS-NAFSA change between $-0.002651 \mathrm{mg}$ and $0.002549 \mathrm{mg}$. Therefore, the measurement errors amplitudes 
TABLE 6: Triaxial accelerometer measurement values with MCS-NAFSA identified parameters.

\begin{tabular}{|c|c|c|c|c|c|c|c|c|c|}
\hline & \multicolumn{3}{|c|}{ Theoretical values $\left(\mathrm{m} / \mathrm{s}^{2}\right)$} & \multicolumn{3}{|c|}{ Calculated values $\left(\mathrm{m} / \mathrm{s}^{2}\right)$} & \multicolumn{3}{|c|}{ Measurement errors (mg) } \\
\hline & $A_{x}^{T}$ & $A_{y}^{T}$ & $A_{z}^{T}$ & $A_{x}^{c}$ & $A_{y}^{c}$ & $A_{z}^{c}$ & $\delta A_{x}$ & $\delta A_{y}$ & $\delta A_{z}$ \\
\hline \multirow{8}{*}{$\begin{array}{l}x \text {-axis point } \\
\text { North }\end{array}$} & 0 & 0 & 9.806550 & 0.000015 & -0.000020 & 9.806536 & 0.001529 & -0.002039 & -0.001427 \\
\hline & 0 & 6.934278 & 6.934278 & 0.000022 & 6.934267 & 6.934263 & 0.002243 & -0.001122 & -0.001529 \\
\hline & 0 & 9.806550 & 0 & 0.000013 & 9.806562 & -0.000018 & 0.001325 & 0.001223 & -0.001835 \\
\hline & 0 & 6.934278 & -6.934278 & -0.000021 & 6.934263 & -6.934296 & -0.002141 & 0.002039 & -0.001835 \\
\hline & 0 & 0 & -9.806550 & -0.000012 & 0.000025 & -9.806564 & -0.001223 & 0.002549 & -0.001427 \\
\hline & 0 & -6.934278 & -6.934278 & -0.000018 & -6.934252 & -6.934268 & -0.001835 & 0.002651 & 0.001020 \\
\hline & 0 & -9.80655 & 0 & -0.000020 & -9.806547 & 0.000018 & -0.002039 & 0.001835 & 0.001835 \\
\hline & 0 & -6.934278 & 6.934278 & 0.000019 & -6.934285 & 6.934288 & 0.001937 & -0.001020 & 0.001020 \\
\hline \multirow{8}{*}{$\begin{array}{l}y \text {-axis point } \\
\text { North }\end{array}$} & 0 & 0 & 9.806550 & 0.000028 & -0.000020 & 9.806540 & 0.002855 & -0.002039 & -0.001020 \\
\hline & -6.934278 & 0 & 6.934278 & -6.934261 & 0.000021 & 6.934269 & 0.001733 & 0.002141 & -0.000917 \\
\hline & -9.80655 & 0 & 0 & -9.806556 & 0.000018 & -0.000026 & -0.000611 & 0.001835 & -0.002651 \\
\hline & -6.934278 & 0 & -6.934278 & -6.934307 & 0.000015 & -6.934298 & -0.001937 & 0.001529 & -0.002039 \\
\hline & 0 & 0 & -9.80655 & -0.000022 & 0.000019 & -9.806560 & -0.002243 & 0.001937 & -0.001020 \\
\hline & 6.934278 & 0 & -6.934278 & 6.934258 & -0.000014 & -6.934267 & -0.002039 & -0.001427 & 0.001121 \\
\hline & 9.80655 & 0 & 0 & 9.806543 & -0.000024 & 0.000008 & -0.001733 & -0.002447 & 0.000815 \\
\hline & 6.934278 & 0 & 6.934278 & 6.934304 & -0.000021 & 6.934288 & 0.001631 & 0.002141 & -0.001020 \\
\hline \multirow{8}{*}{$\begin{array}{l}z \text {-axis point } \\
\text { North }\end{array}$} & 9.806550 & 0 & 0 & 9.806540 & -0.000024 & 0.000018 & -0.001020 & -0.002447 & 0.001835 \\
\hline & 6.934278 & 6.934278 & 0 & 6.934289 & 6.934261 & -0.000022 & 0.001121 & -0.001733 & -0.002202 \\
\hline & 0 & 9.80655 & 0 & 0.000023 & 9.806562 & -0.000020 & 0.002345 & 0.001223 & -0.002039 \\
\hline & -6.934278 & 6.934278 & 0 & -6.934266 & 6.934360 & -0.000013 & 0.001223 & 0.001835 & -0.001325 \\
\hline & -9.80655 & 0 & 0 & -9.806556 & 0.000023 & -0.000016 & -0.000611 & 0.002345 & -0.001631 \\
\hline & -6.934278 & -6.934278 & 0 & -6.934299 & -6.934256 & -0.000024 & -0.001223 & 0.001223 & -0.002447 \\
\hline & 0 & -9.80655 & 0 & -0.000016 & -9.806547 & 0.000020 & -0.001631 & 0.000815 & 0.002039 \\
\hline & 6.934278 & -6.934278 & 0 & 6.934265 & -6.934301 & 0.000025 & -0.001325 & -0.002345 & 0.002549 \\
\hline
\end{tabular}

TABLE 7: Triaxial accelerometer measurement errors statistical results.

\begin{tabular}{lccccc}
\hline & & Mean value $(\mathrm{mg})$ & \multicolumn{3}{c}{ Standard error (mg) } \\
& $\delta A_{x}$ & $\delta A_{y}$ & $\delta A_{z}$ & $\delta A_{x}$ & $\delta A_{y}$ \\
\hline MCS-OAFSA & $-6.845 e-004$ & 0.001688 & -0.001436 & 0.005094 & 0.003731 \\
MCS-NAFSA & $-1.528 e-004$ & $4.459 e-004$ & $-5.8875 e-004$ & 0.001764 & 0.001888 \\
\hline
\end{tabular}

in these figures show that the proposed MCS-NAFSA has smaller measurement errors than the previous MCS-OAFSA in triaxial accelerometer error parameters identification.

Finally, the statistical results in mean values and standard deviations of the triaxial accelerometer measurement errors calculated by MCS-OAFSA and MCS-NAFSA were demonstrated in Table 7 . Both mean values and standard deviations measured by triaxial accelerometer with error parameters identified by MCS-NAFSA are smaller than the error parameters identified by MCS-OAFSA. Therefore, they show the robustness and priority of the proposed MCS-NAFSA in triaxial accelerometer error parameters identification.

7.2. Triaxial Accelerometer-Based SINS Navigation Experiment. In this subsection, a static navigation experiment is carried out by the triaxial accelerometer-based SINS. At the beginning, we install the triaxial accelerometer-based SINS on the marble benchmark to eliminate external disturbances on system positioning precision. Next, start up the SINS and the navigation information (attitude, velocity, position, etc.) which are shown in the monitor. Meanwhile, store the measurement values of triaxial accelerometer and triaxial FOGs and the navigation information for later data processing. After that, make the triaxial accelerometer error parameters identification with OAFSA and the proposed NAFSA with the stored triaxial accelerometer data, respectively. Finally, conduct the navigation mechanization with the OAFSA and NAFSA optimized parameters, respectively. The positioning error calculation formula is given by $[38,39]$

$$
\begin{aligned}
& P_{\text {error }} \\
& =\frac{\sqrt{\left(\text { lat }- \text { lat }_{0}\right)^{2} * R^{2}+\left(\text { long }-\operatorname{long}_{0}\right)^{2} *(R * \cos (\text { lat }))^{2}}}{1851.8518}
\end{aligned}
$$


TABLE 8: Static positioning results of three different methods.

\begin{tabular}{lccc}
\hline Methods & Conventional calibration & OAFSA identification & NAFSA identification \\
\hline 24-hour position error (nmile) & 4.4895 & 4.4988 & 4.2550 \\
\hline
\end{tabular}

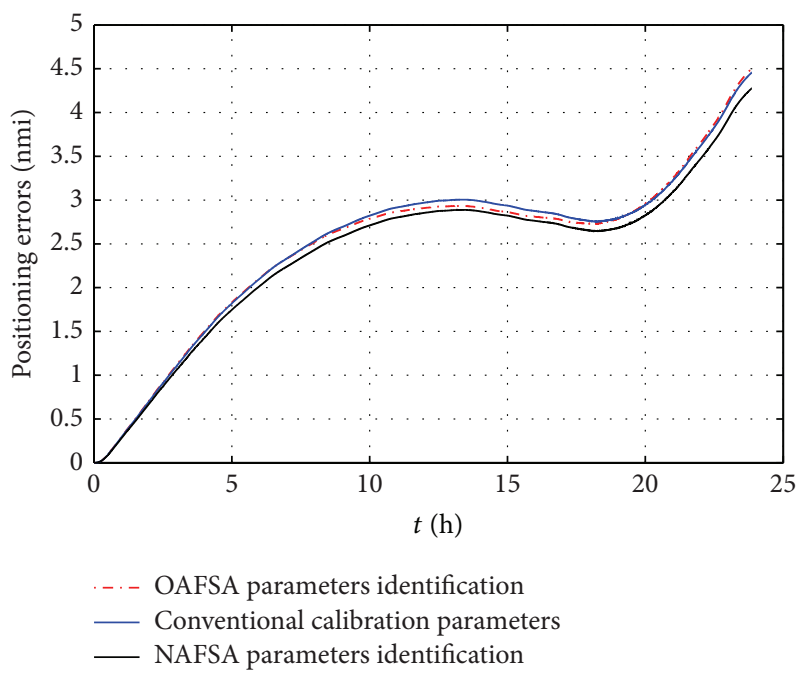

FIGURE 10: The comparison of positioning errors with three methods.

where long $_{0}$ and lat a $_{0}$ are the initial longitude and latitude of the vehicle, long and lat are the calculated longitude and latitude, and $R$ denotes the radius of Earth.

Figure 10 shows a comparison of positioning errors with the conventional calibration method and the OAFSA and the NAFSA on triaxial accelerometer error parameters in 24-hour static navigation experiment. From Figure 10, we can conclude that the red dotted positioning error curve represents the OAFSA-identified triaxial accelerometer error parameters identification results. And also the blue dotted curve represents the conventional high-precision turntable triaxial accelerometer calibration method. Both curves have positioning precision of $4.5 \mathrm{nmiles}$ in 24 -hour static navigation experiment, which shows that the OAFSA could substitute the conventional calibration method without using high-precision turntable. Furthermore, it is worth noting that the black solid curve in Figure 10 represents positioning precision of the proposed NAFSA on triaxial accelerometer error parameters identification. The black solid curve's tendency demonstrates that, after 5-hour navigation, the positioning error is lower than the former two methods and the positioning precision is about $0.3 \mathrm{nmiles}$ better than the OAFSA in one-day navigation experiment.

The corresponding numerical results of static positioning errors with the three different methods are shown in Table 8. Both the conventional calibration method and the OAFSA have about 4.5-nmile positioning error in 24 hours. The proposed NAFSA has 4.255 -nmile positioning error at the same time.

Therefore, we can summarize that the proposed NAFSA has advantages in workload and costs compared to the conventional calibration method. Moreover, the NAFSA has better performances in long-term navigation precision and has been more acceptable for actual engineering applications with lower computation complexity and faster convergence rate.

\section{Conclusion}

After the triaxial accelerometer-based SINS operated for a period of time, the triaxial accelerometer would be vulnerable by the working environmental disturbances, such as gravitational field, magnetic field, and thermal field. These exterior disturbances could influence the triaxial accelerometer error parameters' stability directly or indirectly. Even though some measures are taken to eliminate these effects, high-precision navigation application is far from enough.

The research work in this paper is based on one of the swarm intelligence algorithms, artificial fish swarm algorithm, mainly on its optimization and improvement algorithm for triaxial accelerometer error parameters identification. The proposed NAFSA has the advantages of lower computational complexity and higher convergence rate than the OAFSA during optimization process. And it also has lesser workload and costs requirements than the conventional triaxial accelerometer error parameters calibration method. Furthermore, the proposed method could implement shorter recalibration interval time with higher precision in some harness application environment.

Both the 24-position triaxial accelerometer verification experiment and the triaxial accelerometer-based SINS navigation experiment results show that when the triaxial accelerometer-based SINS is in navigation condition, the proposed NAFSA on triaxial accelerometer error parameters identification could implement the SINS navigation process rapidly and accurately. Moreover, the NAFSA-identified triaxial accelerometer error parameters have better environment adaptive ability, which means higher positioning accuracy and better tracking performance. Therefore, the proposed NAFSA has better ability than the conventional calibration method and the OAFSA in triaxial accelerometer error parameters identification applications.

However, the AFSA on triaxial accelerometer error parameters identification is only in exploration phase and all the navigation experiments are based on the stored data. So our work for next stage is to realize the algorithm in real-time navigation.

\section{Conflict of Interests}

The authors declare that there is no conflict of interests regarding the publication of this paper. 


\section{Acknowledgments}

Lianwu Guan is sponsored by the China Scholarship Council (CSC) for his joint Ph.D. research training program at the Queen's University, Kingston, ON, Canada. This research work is supported by the Ministry of Science and Technology of China (no. 2014DFR10010) and sponsored by the National Natural Science Foundation of China (51309059). The authors also would like to thank Dr. Jonathan Wylie for helping to edit the language of the paper and anonymous reviewers for improving this paper.

\section{References}

[1] X.-L. Li, Z.-J. Shao, and J.-X. Qian, "An optimizing method based on autonomous animate: fish swarm algorithm," System Engineering Theory and Practice, vol. 11, pp. 32-38, 2002.

[2] X.-L. Li, F. Lu, G.-H. Tian, and J.-X. Qian, "Applications of artificial fish school algorithm in combinatorial optimization problems," Journal of Shan Dong University (Engineering Science), vol. 34, no. 5, pp. 64-67, 2004.

[3] M. Tuba, N. Bacanin, and N. Stanarevic, "Multithreaded implementation and performance of a modified artificial fish swarm algorithm for unconstrained optimization," International Journal of Mathematics and Computers in Simulation, vol. 7, no. 3, pp. 215-222, 2013.

[4] W. Shen, X. Guo, C. Wu, and D. Wu, "Forecasting stock indices using radial basis function neural networks optimized by artificial fish swarm algorithm," Knowledge-Based Systems, vol. 24, no. 3, pp. 378-385, 2011.

[5] R. Song, X. Chen, C. Shen, and H. Zhang, "Modeling FOG drift using back-propagation neural network optimized by artificial fish swarm algorithm," Journal of Sensors, vol. 2014, Article ID 273043, 6 pages, 2014.

[6] P. Vannoorenberghe and G. Flouzat, "A belief-based pixel labelling strategy for medical and satellite image segmentation," in Proceedings of the IEEE International Conference on Fuzzy Systems, pp. 1093-1098, July 2006.

[7] A. K. C. Wong and G. C. L. Li, "Simultaneous pattern and data clustering for pattern cluster analysis," IEEE Transactions on Knowledge and Data Engineering, vol. 20, no. 7, pp. 911-923, 2008.

[8] T. Fei, L. Zhang, Y. Li, Y. Yang, and F. Wang, "The artificial fish swarm algorithm to solve traveling salesman problem," in Proceedings of International Conference on Computer Science and Information Technology, vol. 255 of Advances in Intelligent Systems and Computing, pp. 679-685, Springer, New Delhi, India, 2014.

[9] Z. Cheng and X. Hong, "PID controller parameters optimization based on artificial fish swarm algorithm," in Proceedings of the IEEE 5th International Conference on Intelligent Computation Technology and Automation (ICICTA '12), pp. 265-268, January 2012.

[10] H.-C. Tsai and Y.-H. Lin, "Modification of the fish swarm algorithm with particle swarm optimization formulation and communication behavior," Applied Soft Computing, vol. 11, no. 8, pp. 5367-5374, 2011.

[11] M. Chih, L.-L. Yeh, and F.-C. Li, "Particle swarm optimization for the economic and economic statistical designs of the $\bar{X}$ control chart," Applied Soft Computing Journal, vol. 11, no. 8, pp. 5063-5067, 2011.
[12] M. Chih, C.-J. Lin, M.-S. Chern, and T.-Y. Ou, "Particle swarm optimization with time-varying acceleration coefficients for the multidimensional knapsack problem," Applied Mathematical Modelling, vol. 38, no. 4, pp. 1338-1350, 2014.

[13] M. Chih, "Self-adaptive check and repair operator-based particle swarm optimization for the multidimensional knapsack problem," Applied Soft Computing, vol. 26, pp. 378-389, 2015.

[14] W.-C. Yeh, Y.-C. Lin, Y. Y. Chung, and M. Chih, "A particle swarm optimization approach based on monte carlo simulation for solving the complex network reliability problem," IEEE Transactions on Reliability, vol. 59, no. 1, pp. 212-221, 2010.

[15] W. H. Lim and N. A. Mat Isa, "Two-layer particle swarm optimization with intelligent division of labor," Engineering Applications of Artificial Intelligence, vol. 26, no. 10, pp. 23272348, 2013.

[16] I. Idris, A. Selamat, and S. Omatu, "Hybrid email spam detection model with negative selection algorithm and differential evolution," Engineering Applications of Artificial Intelligence, vol. 28, pp. 97-110, 2014.

[17] D. Yazdani, B. Saman, A. Sepas-Moghaddam, F. MohammadKazemi, and M. Reza Meybodi, "A new algorithm based on improved artificial fish swarm algorithm for data clustering," International Journal of Artificial Intelligence, vol. 11, no. 13, pp. $1-29,2013$.

[18] Y.-B. Gao, T.-J. Wang, G.-C. Li, and Y.-P. Shao, "Error parameter identification of accelerometer based on AFSA," Systems Engineering and Electronics, vol. 35, no. 9, pp. 1933-1939, 2013.

[19] Y.-B. Gao, L.-W. Guan, T.-J. Wang, and X.-D. Cong, "Research on the calibration of FOG based on AFSA," in Proceedings of the 10th IEEE International Conference on Mechatronics and Automation (ICMA '13), pp. 412-417, August 2013.

[20] Y.-B. Gao, L.-W. Guan, and T.-J. Wang, "Optimal artificial fish swarm algorithm for the field calibration on marine navigation," Measurement, vol. 50, no. 1, pp. 297-304, 2014.

[21] M. Perlmutter and L. Robin, "High-performance, low cost inertial MEMS: a market in motion!", in Proceedings of the IEEE/ION Position, Location and Navigation Symposium (PLANS '12), pp. 225-229, Myrtle Beach, SC, USA, April 2012.

[22] V. Renaudin, C. Combettes, and F. Peyret, "Quaternion based heading estimation with handheld MEMS in indoor environments," in Proceedings of the IEEE/ION Position, Location and Navigation Symposium (PLANS '14), pp. 645-656, IEEE, Monterey, Calif, USA, May 2014.

[23] L. Luo, "Attitude angular measurement system based on MEMS accelerometer," in 7th International Symposium on Advanced Optical Manufacturing and Testing Technologies: Large Mirrors and Telescopes, vol. 9280 of Proceedings of SPIE, p. 928016, Harbin, China, September 2014.

[24] S.-H. P. Won and F. Golnaraghi, "A triaxial accelerometer calibration method using a mathematical model," IEEE Transactions on Instrumentation and Measurement, vol. 59, no. 8, pp. 2144-2153, 2010.

[25] G. A. Aydemir and A. Saranli, "Characterization and calibration of MEMS inertial sensors for state and parameter estimation applications," Measurement, vol. 45, no. 5, pp. 1210-1225, 2012.

[26] T.-J. Wang, Y.-B. Gao, G.-C. Li, and Q. Lin, "FOG random drift modeling by artificial fish swarm algorithm," Journal of Chinese Inertial Technology, vol. 20, no. 3, pp. 358-362, 2012.

[27] X.-D. Yu, Y.-N. Xu, G. Wei, and X. W. Long, “Temperature compensation method for bias of ring laser gyroscope based on artificial fish swarm algorithm," Infrared and Laser Engineering, vol. 43, no. 1, pp. 81-87, 2014. 
[28] Q. Yang and Y. Chen, "Monte Carlo methods for reliability evaluation of linear sensor systems," IEEE Transactions on Reliability, vol. 60, no. 1, pp. 305-314, 2011.

[29] L.-G. Wang and Q.-H. Shi, "Parameters analysis of artificial fish swarm algorithm," Computer Engineering, vol. 36, no. 24, pp. 169-171, 2010.

[30] Y. Shi and R. Eberhart, "A modified particle swarm optimizer," in Proceedings of the IEEE International Conference on World Congress on Computational Intelligence: Evolutionary Computation Proceedings, pp. 69-73, May 1998.

[31] Z. F. Syed, P. Aggarwal, C. Goodall, X. Niu, and N. El-Sheimy, "A new multi-position calibration method for MEMS inertial navigation systems," Measurement Science and Technology, vol. 18, no. 7, pp. 1897-1907, 2007.

[32] H. Zhang, Y. Wu, M. Wu, X. Hu, and Y. Zha, "A multiposition calibration algorithm for inertial measurement units," in Proceedings of the AIAA Guidance, Navigation and Control Conference and Exhibit, Honolulu, Hawaii, USA, August 2008.

[33] I. Frosio, F. Pedersini, and N. A. Borghese, "Autocalibration of MEMS accelerometers," IEEE Transaction on Instrument and Measurement, vol. 58, pp. 2034-2041, 2009.

[34] X. Li and Z. Li, "A new calibration method for tri-axial field sensors in strap-down navigation systems," Measurement Science and Technology, vol. 23, no. 10, Article ID 105105, pp. 19, 2012.

[35] Q. Cai, N. Song, G. Yang, and Y. Liu, "Accelerometer calibration with nonlinear scale factor based on multi-position observation," Measurement Science and Technology, vol. 24, no. 10, Article ID 105002, 2013.

[36] W. T. Song and B. W. Schmeiser, "Omitting meaningless digits in point estimates: the probability guarantee of leading-digit rules," Operations Research, vol. 57, no. 1, pp. 109-117, 2009.

[37] A. M. Law, Simulation Modeling and Analysis, McGraw-Hill, Boston, Mass, USA, 4th edition, 2007.

[38] P. G. Savage, "Strapdown inertial navigation integration algorithm design part 2: velocity and position algorithms," Journal of Guidance, Control, and Dynamics, vol. 21, no. 2, pp. 208-221, 1998.

[39] O. S. Salychev, Applied Inertial Navigation: Problems and Solutions, BMSTU Press, Moscow, Russia, 2004. 

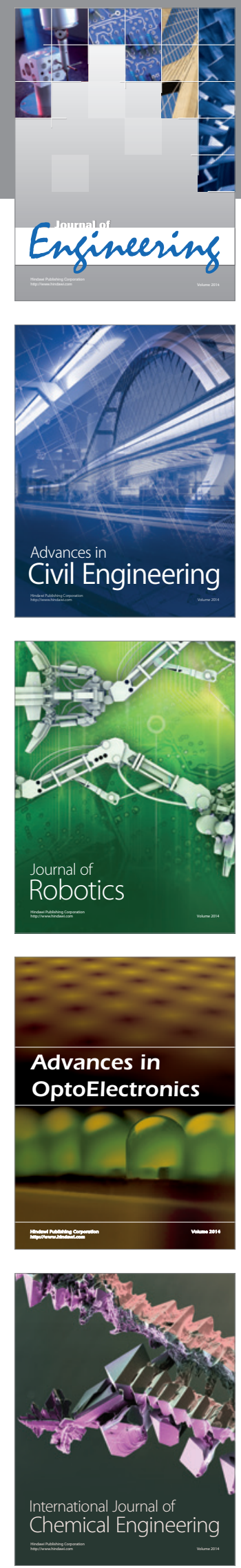

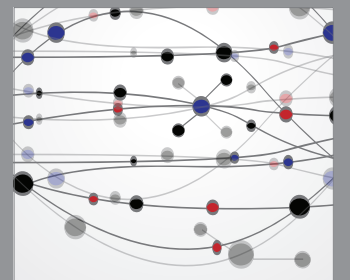

The Scientific World Journal
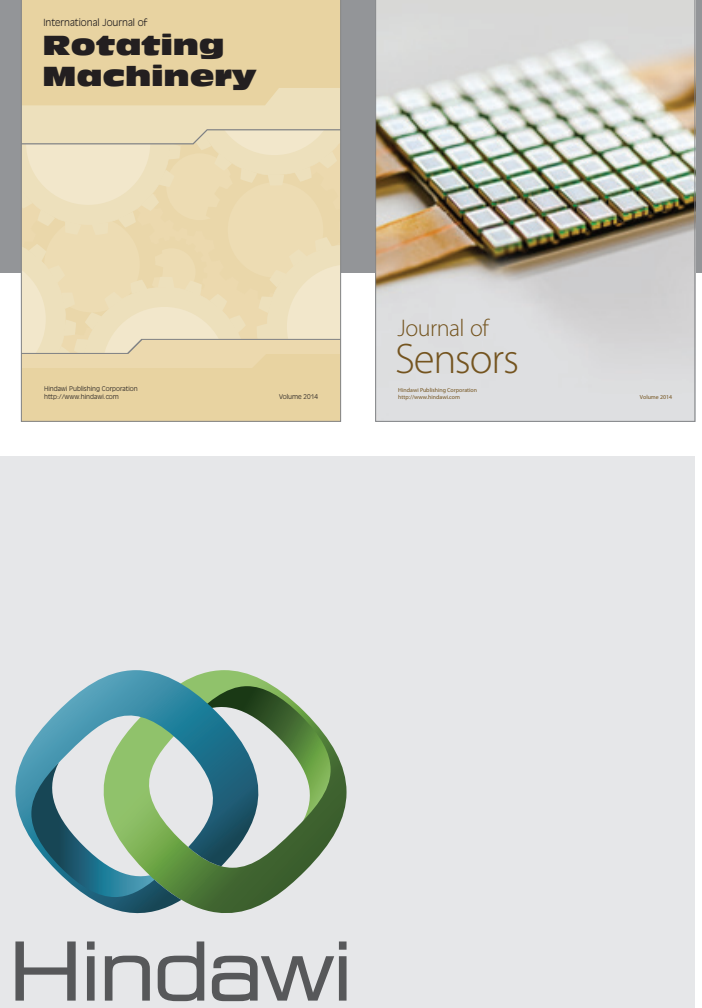

Submit your manuscripts at http://www.hindawi.com
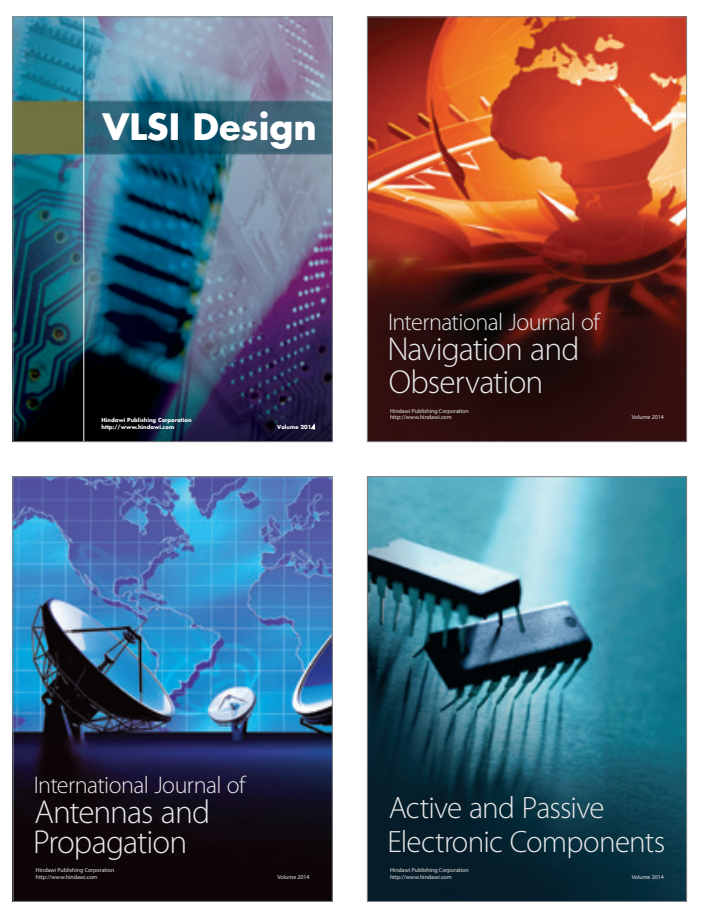
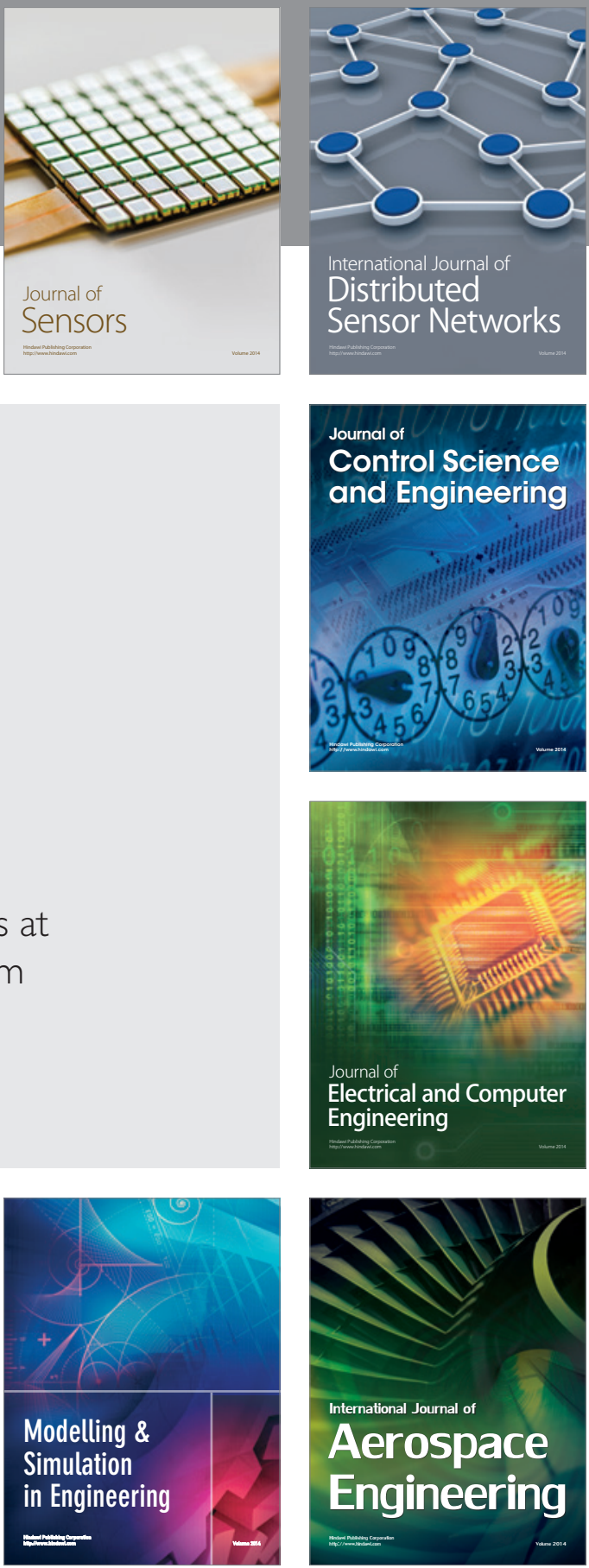

Journal of

Control Science

and Engineering
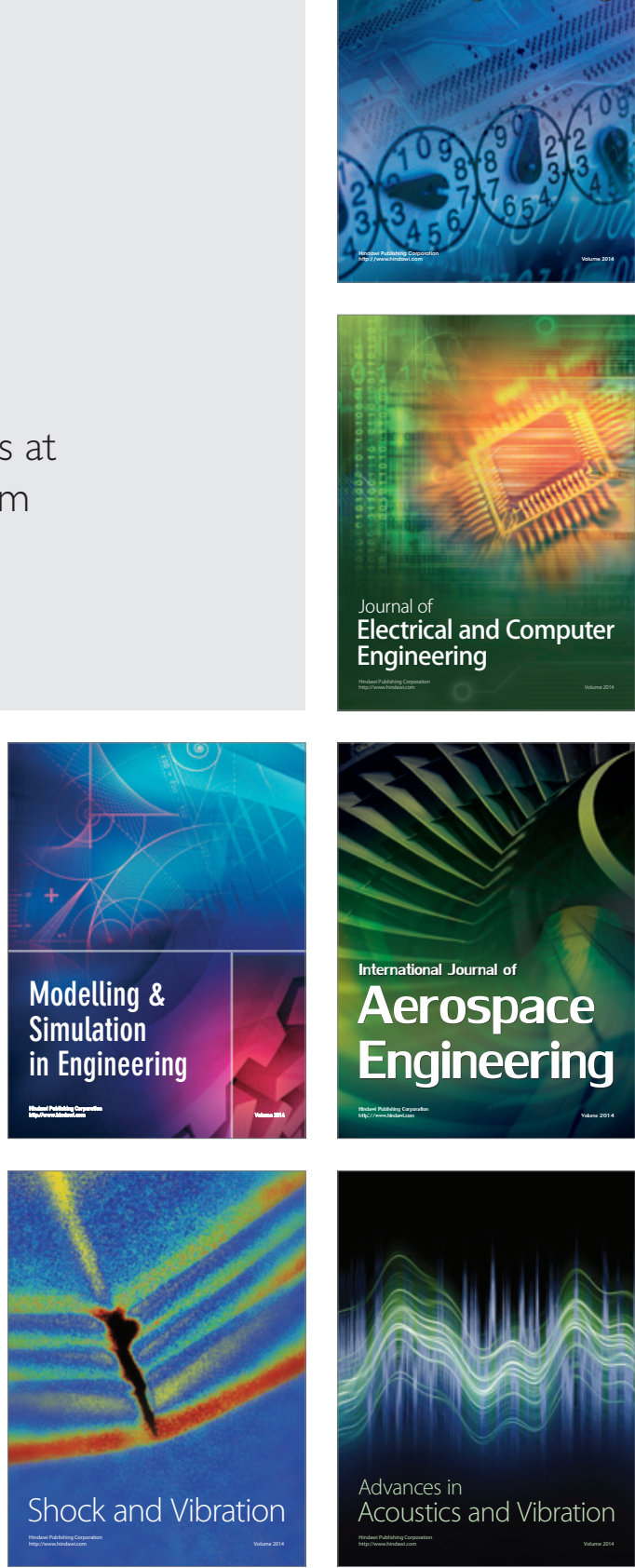